Moshe Marcus · Laurent Véron

\title{
Capacitary estimates of positive solutions of semilinear elliptic equations with absorbtion
}

Received February 15, 2004

\begin{abstract}
Let $\Omega$ be a bounded domain of class $C^{2}$ in $\mathbb{R}^{N}$ and let $K$ be a compact subset of $\partial \Omega$. Assume that $q \geq(N+1) /(N-1)$ and denote by $U_{K}$ the maximal solution of $-\Delta u+u^{q}=0$ in $\Omega$ which vanishes on $\partial \Omega \backslash K$. We obtain sharp upper and lower estimates for $U_{K}$ in terms of the Bessel capacity $C_{2 / q, q^{\prime}}$ and prove that $U_{K}$ is $\sigma$-moderate. In addition we describe the precise asymptotic behavior of $U_{K}$ at points $\sigma \in K$, which depends on the "density" of $K$ at $\sigma$, measured in terms of the capacity $C_{2 / q, q^{\prime}}$.
\end{abstract}

\section{Introduction}

Let $\Omega \subset \mathbb{R}^{N}$ be a bounded domain whose boundary is of class $C^{2}$ and let $q>1$. If $\mu$ is a Radon measure on $\Sigma:=\partial \Omega$, the problem

$$
-\Delta u+u^{q}=0 \quad \text { in } \Omega, \quad u=\mu \quad \text { on } \Sigma,
$$

has a solution if and only if $\mu$ vanishes on sets of $C_{2 / q, q^{\prime}}$ capacity zero, which is equivalent to $\mu \in W_{+}^{-2 / q, q}(\Sigma)$ (see [15]). The solution is unique and will be denoted by $u_{\mu}$. Following Dynkin and Kuznetsov [7], a positive solution $u \in C^{2}(\Omega)$ of the equation

$$
-\Delta u+u^{q}=0 \quad \text { in } \Omega
$$

is called $\sigma$-moderate if there exists an increasing sequence of positive Radon measures $\mu_{n}$ on $\Sigma$ such that the sequence $\left\{u_{\mu_{n}}\right\}$ converges to $u$. Similarly a measure $\mu \in\left(\mathcal{B}_{r}(\Sigma)\right)_{+}$ $\left(\mathcal{B}_{r}=\right.$ space of regular Borel measures, not necessarily bounded $)$ is called $\sigma$-moderate if it is the limit of an increasing sequence of measures belonging to $W_{+}^{-2 / q, q}(\Sigma)$. The space of $\sigma$-moderate measures will be denoted by $\mathcal{B}_{+}^{q}(\Sigma)$. If $1<q<q_{c}=(N+1) /(N-1)$ a singleton has positive $C_{2 / q, q^{\prime}}$ capacity so that every bounded Borel measure is in $W_{+}^{-2 / q, q}(\Sigma)$. If $q \geq q_{c}$ a singleton has $C_{2 / q, q^{\prime}}$ capacity zero. The number $q_{c}$ is called the critical value of the exponent for (1.1).

This research was supported by RTN contract No. HPRN-CT-2002-00274.

M. Marcus: Department of Mathematics, Israel Institute of Technology-Technion, 32000 Haifa, Israel; e-mail: marcusm@math.technion.ac.il

L. Véron: Département de Mathématiques, Faculté des Sciences et Techniques, Université de Tours, 37200 Tours, France; e-mail: veronl@univ-tours.fr 
A central question in the study of 1.2$]$ is the behavior of solutions in the neighborhood of boundary singularities. Indeed this pertains to the essence of the boundary value problem (1.1) which naturally includes singular boundary data. To make the notion of singularity more precise we introduce the following notation. Let

$$
\mathcal{K}_{q}(\partial \Omega)=\left\{K \subset \partial \Omega: K \text { compact, } C_{2 / q, q^{\prime}}(K)>0\right\} .
$$

For every $K \in \mathcal{K}_{q}(\partial \Omega)$ we denote by $U_{K}$ the maximal solution of 11.2 which vanishes outside $K$. It is known that $U_{K}$ is not the trivial solution. Let $u$ be a positive solution of (1.2). If there exists a set $K \in \mathcal{K}_{q}(\partial \Omega)$ such that $U_{K} \leq u$ we say that $u$ is singular on $K$.

In the subcritical case, there exists a minimal singularity from which all other singularities can be constructed, namely, the point singularity. The asymptotic behavior of solutions at a point singularity on the boundary was investigated by Gmira and Véron [9]. Several years later, these estimates constituted one of the main ingredients in the study of the general boundary value problem 11.1 in the subcritical case (see Marcus and Véron [12] and [13]). In the later work it was shown that every positive solution has a boundary trace in $\left(\mathcal{B}_{r}(\Sigma)\right)_{+}$, and conversely, for every positive $\mu \in \mathcal{B}_{r}(\Sigma)$ there exists a unique solution of the equation whose boundary trace is $\mu$. In the special case $q=N=2$ this result was previously established by Le Gall [11] by probabilistic methods.

In the supercritical case, such a minimal singularity does not exist, and this makes the situation enormously more complicated. In order to relate singular solutions to boundary singular sets, let us consider the case $\Omega=B_{1}(0)$. We say that two sets $K_{1}, K_{2} \in \mathcal{K}_{q}(\partial \Omega)$ are $q$-related if there exists a rotation $T$ such that $T\left(K_{1}\right) \cap K_{2} \in \mathcal{K}_{q}(\partial \Omega)$. Equivalently, the singular solutions $U_{K_{1}}, U_{K_{2}}$ are $q$-related if there exists a rotation $T$ and a positive solution $u$ such that $u \leq \min \left(U_{T\left(K_{1}\right)}, U_{K_{2}}\right)$.

The characterization of the boundary data and its interpretation, i.e. the sense in which the boundary data is attained, is another problem whose treatment is more complicated in the supercritical case. The space $\left(\mathcal{B}_{r}\right)_{+}$is no longer sufficient in this case. Although it is still true that problem (1.1) has a solution for every $\mu \in\left(\mathcal{B}_{r}(\Sigma)\right)_{+}$, uniqueness fails. This fact was observed by Le Gall in 1997. Following this observation Dynkin and Kuznetsov [7] introduced the class of $\sigma$-moderate solutions and showed that, in this class, problem (1.1) has a unique solution. In a remarkable paper, Mselati [17] proved that, if $q=2$, all solutions are $\sigma$-moderate. A related result, applying to arbitrary $q>1$, was recently established by Marcus and Véron [16]: For every compact set $K \in \mathcal{K}_{q}(\partial \Omega)$ the maximal solution $U_{K}$ is $\sigma$-moderate.

In the case $q=2$ this result was previously established by Mselati, in the paper quoted above, and played a key role in the derivation of his main result. His proof used mainly probabilistic techniques and in particular the notion of Brownian snake introduced by Le Gall. Our proof employs purely analytic methods. (The proof was sketched in [16] and it is given in detail in the present paper.) Following this, Dynkin [3]-[5] announced the extension of Mselati's main result to the range $1<q \leq 2$ by employing probabilistic methods developed in joint work with Kuznetsov (see [8] and references therein) and the above mentioned result of [16]. He also provided an alternative, probabilistic proof of the latter result which, however, applies only to the range $1<q \leq 2$. These results are presented in a forthcoming book [6]. 
We turn to the description of our main results. The first of these provides sharp estimates, from above and below, for maximal solutions $U_{K}$ of (1.2). Besides $U_{K}$ we also consider the solution $u_{K}$ defined as the supremum of all solutions of (1.2) whose boundary trace is a bounded measure supported in $K$. This is in fact the maximal $\sigma$-moderate solution vanishing on $\partial \Omega \backslash K$.

Theorem 1.1. Let $q \geq q_{c}$ and $K \in \mathcal{K}_{q}(\partial \Omega)$. Then there exist positive constants $c_{1}, c_{2}$ depending only on $q, N$ and $\Omega$ such that the following inequalities hold for every $x \in \Omega$ :

$$
\begin{gathered}
U_{K}(x) \leq c_{1} \rho(x) \rho_{K}(x)^{-1-2 /(q-1)} C_{2 / q, q^{\prime}}\left(K / \rho_{K}(x)\right), \\
U_{K}(x) \geq u_{K}(x) \geq c_{2} \rho(x) \sum_{m=-\ell(\Omega)}^{\infty} r_{m}^{-1-2 /(q-1)} C_{2 / q, q^{\prime}}\left(K_{m}(x) / r_{m}\right),
\end{gathered}
$$

where $\ell(\Omega)$ is a number which depends only on diam $\Omega$ and

$$
\begin{gathered}
\rho(x):=\operatorname{dist}(x, \partial \Omega), \quad \rho_{K}(x):=\operatorname{dist}(x, K), \\
K_{m}(x):=\left\{y \in K: r_{m+1} \leq|x-y| \leq r_{m}\right\}, \quad r_{m}:=2^{-m} \quad \forall m \in \mathbb{Z} .
\end{gathered}
$$

It is easy to see that 1.3 implies

$$
U_{K}(x) \leq c_{3} \rho(x) \sum_{m=-\ell(\Omega)}^{\infty} r_{m}^{-1-2 /(q-1)} C_{2 / q, q^{\prime}}\left(K_{m}(x) / r_{m}\right) .
$$

Therefore the inequalities are sharp. In the case $q=2$, [1.3] was obtained by Mselati [17] employing a mixture of analytic and probabilistic techniques which apply only to this case. Our proof, which is purely analytic, is based mainly on two techniques. The first involves a linear lifting from $C(\partial \Omega)$ to $C^{0,1}(\Omega)$, somewhat similar to the lifting introduced by the authors in [15]. Using this lifting we obtain an integral capacitary estimate from above. The second technique involves an (apparently) new estimate for Poisson kernels (see Appendix C) and is used in order to pass from this integral estimate to the pointwise estimate (1.3). The lower estimate obtained by Mselati [17], for $q=2$, was expressed in probabilistic terms and it is not directly comparable to (1.4). In the case of solutions with interior singularities, i.e. singularities in the domain $\Omega$, capacitary estimates were obtained by Labutin [10] for all $q \geq N /(N-2)$, the latter being the critical value of the exponent for the equation $-\Delta u+u^{q}=\mu$. Those estimates are sharp for $q>N /(N-2)$, but not in the critical case. Our proof of the lower estimate 1.4 is inspired by some of the techniques of [10].

Next we present several applications of Theorem 1.1 As a first application we derive the identity

$$
U_{K}=u_{K}
$$

which shows that $U_{K}$ is $\sigma$-moderate.

The second application is an estimate of $U_{K}(x)$ as $x \in \Omega$ tends (non-tangentially) to a point $\sigma \in K$. Here the behavior of $U_{K}$ depends on the "capacitary distribution" of $K$ in the neighborhood of $\sigma$, which we define by

$$
\theta_{K}(t ; \sigma):=C_{2 / q, q^{\prime}}\left(\frac{1}{t}\left(K \cap B_{t}(\sigma)\right)\right), \quad 0<t<1 .
$$


Now, for every $a \geq 1$, we have

$$
\begin{aligned}
\frac{1}{c(a)} \int_{s}^{1} t^{-1-2 /(q-1)} \theta_{K}(t ; \sigma) \frac{d t}{t} & \leq \frac{U_{K}(x)}{|x-\sigma|} \\
& \left.\leq c(a) \int_{s}^{1} t^{-1-2 /(q-1)} \theta_{K}(t ; \sigma)\right) \frac{d t}{t}+O(1)
\end{aligned}
$$

for all $x \in \Omega$ with $s=|x-\sigma| \leq a \rho(x)$. The constant $c(a)$ depends only on $a, q, N, \Omega$. Evidently these too are sharp estimates. Further we show that if $K$ is $\left(2 / q, q^{\prime}\right)$-thick at $\sigma$ (for the definition of this term see [1] or Section 4 below) then

$$
\int_{0}^{1} t^{-1-2 /(q-1)} \theta_{K}(t ; \sigma) \frac{d t}{t}=\infty .
$$

Therefore at such a point

$$
U_{K}(x) /|x-\sigma| \rightarrow \infty \text { as } x \rightarrow \sigma \text { non-tangentially. }
$$

It is known that $K$ is $\left(2 / q, q^{\prime}\right)$-thick everywhere in $K$, with the possible exception of a set

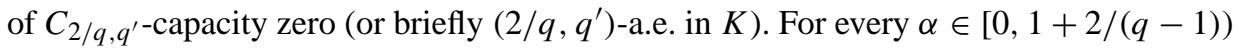
and $\sigma_{0} \in \partial \Omega$ one can construct a set $K \in \mathcal{K}_{q}(\partial \Omega)$ such that $\theta_{K}\left(t ; \sigma_{0}\right) \approx t^{\alpha}$. For such $K$, (1.8) holds at $\sigma_{0}$ and we have

$$
U_{K}(x) \approx\left|x-\sigma_{0}\right|^{\alpha-2 /(q-1)} \quad \text { as } x \rightarrow \sigma_{0} \text { non-tangentially. }
$$

Thus $U_{K}(x)$ may remain bounded or blow up at any rate not larger than $s^{-2 /(q-1)}$ as $x$ tends non-tangentially to a point where 1.8 holds.

On the other hand, we show that at every $\left(2 / q, q^{\prime}\right)$-thick point of $K, U_{K}$ blows up in an integral sense, at the maximal rate. More precisely we prove:

If $K$ is $\left(2 / q, q^{\prime}\right)$-thick at $\sigma$ then

$$
\int_{0}^{1} U_{K}(\Gamma(t))^{q-1} t d t=\infty
$$

for every curve $\Gamma \in \operatorname{Lip}([0,1], \Omega \cup\{\sigma\})$ such that $\Gamma(0)=\sigma$ and $0<|\Gamma(t)-\sigma| \leq$ $a \rho(\gamma(t))$ for some $a \geq 1$ and every $t \in(0,1]$.

Incidentally, this fact and the previous remarks imply that 1.8 may hold at a point $\sigma$ where $K$ is not $\left(2 / q, q^{\prime}\right)$-thick.

The plan of the paper is as follows: Section 2 is devoted to the proof of the upper estimate (1.3). In Section 3 we present the proof of the lower estimate (1.4). Section 4 is devoted to the proof of (1.6), (1.7), (1.9) and related results. In Appendix A and B we derive some elementary estimates involving capacity which are used throughout the paper. Appendix $\mathrm{C}$ is devoted to an estimate of Poisson kernels which is used in Section 2. This estimate may be of interest in itself.

\section{Boundary singularities: estimates from above}

We start with some basic notations. $C_{\alpha, p}(0<\alpha, 1<p<\infty)$ denotes Bessel capacity in $\mathbb{R}^{N-1}$ or alternatively on a smooth manifold such as $\Sigma=\partial \Omega$. If $A \subset \Sigma$ then $C_{\alpha, p}(A)$ 
denotes the capacity of $A$ relative to $\Sigma$ and, if $\gamma>0, C_{\alpha, p}(\gamma A)$ denotes the capacity of $\gamma A$ relative to $\gamma \Sigma$. Further, for any set $E \subset \mathbb{R}^{N}$ and $\beta>0$, we write

$$
\rho_{E}(x):=\operatorname{dist}(x, E), \quad(E)^{\beta}:=\left\{x \in \mathbb{R}^{N}: \rho_{E}(x)<\beta\right\} .
$$

For any domain $D$ and $\beta_{1}, \beta_{2}>0$, we define

$$
\dot{\rho}_{D}(x):=\left\{\begin{aligned}
\rho_{\partial D}(x) & \text { if } x \in D, \\
-\rho_{\partial D}(x) & \text { if } x \in D^{c},
\end{aligned}\right.
$$

and

$$
D_{\beta_{1}, \beta_{2}}:=\left\{x \in \mathbb{R}^{N}: \beta_{1}<\dot{\rho}_{D}(x)<\beta_{2}\right\}, \quad D_{\beta}:=D_{0, \beta}, \quad D_{\beta}^{e}:=D_{-\beta, 0} .
$$

Since $\Sigma=\partial \Omega$ is a closed, compact manifold of class $C^{2}$ there exists a positive number $\beta^{i}(\Omega)\left(\operatorname{resp} \beta^{e}(\Omega)\right)$ such that for every $x$ in the closure of $\Omega_{\beta^{i}}\left(\right.$ resp. $\Omega_{\beta^{e}}^{e}$ ) there exists a unique point $\sigma_{\Sigma}(x) \in \Sigma$ for which $\left|x-\sigma_{\Sigma}(x)\right|=\rho_{\Sigma}(x)$. Furthermore the mapping

$$
\Pi: \Omega_{-\beta^{e}, \beta^{i}} \rightarrow\left(-\beta^{e}, \beta^{i}\right) \times \Sigma \quad \text { given by } \Pi(x)=\left(\dot{\rho}_{\Omega}(x), \sigma_{\Sigma}(x)\right)
$$

is a $C^{2}$ diffeomorphism. We set $\beta_{0}(\Omega):=\min \left(\beta^{i}(\Omega), \beta^{e}(\Omega)\right.$ ). For every $x \in \Omega_{-\beta^{e}, \beta^{i}}$, denote by $v_{\Omega}(x)$ the outward unit normal (with respect to $\Omega$ ) at $\sigma_{\Sigma}(x)$. Clearly $\nu_{\Omega} \in C^{1}$. To simplify the notation, we drop the subscript in $\rho_{\Sigma}$ and $\sigma_{\Sigma}$.

If $E \subset \Sigma$ and $a \geq 1$, put

$$
C_{a}^{\Omega}(E)=\left\{x \in \Omega: \rho_{E}(x) \leq a \rho(x)\right\}
$$

and $C_{a}^{\Omega}(\xi):=C_{a}^{\Omega}(E)$ if $E=\{\xi\}$ is a singleton.

Throughout this paper $c$ denotes a positive constant which depends only on $q, N, \Omega$ and the choice of $\beta_{0}$. The value of the constant may change from one occurrence to another. The notation $X \approx Y$ means $(1 / c) X \leq Y \leq c X$ for some constant $c$. If this constant depends on an additional parameter, say $a$, we write $X \stackrel{a}{\approx} Y$. Finally, we shall assume, without further mention, that $q \geq q_{c}=(N+1) /(N-1)$ and $K$ is a compact subset of $\Sigma$.

Our upper estimate is presented in the following theorem.

Theorem 2.1. Let $u$ be a positive solution of 1.2 which vanishes on $K^{c}:=\Sigma \backslash K$. Then there exists a constant $c$, independent of $u$ and $K$, such that

$$
u(x) \leq c \rho(x) \rho_{K}(x)^{-1-2 /(q-1)} C_{2 / q, q^{\prime}}\left(K / \rho_{K}(x)\right)
$$

for every $x \in \Omega$.

Remark. For $\xi \in \mathbb{R}^{N}$ and $r>0$ put

$$
[K / r]_{\xi}:=\frac{K-\xi}{r}+\xi=\frac{K}{r}+\frac{r-1}{r} \xi .
$$

Clearly $C_{2 / q, q^{\prime}}\left([K / r]_{\xi}\right)=C_{2 / q, q^{\prime}}(K / r)$. 
A key element in the proof of the theorem is a lifting from $C(\partial \Omega)$ into $C(\bar{\Omega}) \cap C^{0,1}(\Omega)$ which is a modification of the lifting used in [15]. It is defined as follows.

Definition 2.2. Let $\eta \in C^{2}(\Sigma)$ and let $H_{\eta}$ be the solution of the initial value problem

$$
\begin{array}{ll}
\frac{\partial H}{\partial \rho}=\Delta_{\Sigma} H & \text { in } \mathbb{R}_{+} \times \Sigma, \\
H(0, \cdot)=\eta(\cdot) & \text { in } \Sigma .
\end{array}
$$

Let $\varphi$ be the solution of the problem

$$
-\Delta \varphi=1 \quad \text { in } \Omega, \quad \varphi=0 \text { on } \partial \Omega .
$$

Let $R=R_{\eta}$ be the lifting given by

$$
R_{\eta}(x)= \begin{cases}H_{\eta}\left(\varphi(x)^{2}, \sigma(x)\right) & \forall x \in \bar{\Omega}_{\beta_{0}}, \\ V_{\eta} & \forall x \in \Omega \backslash \Omega_{\beta_{0}},\end{cases}
$$

where $V_{\eta}$ is the harmonic function in $\Omega_{\beta_{0}}^{\prime}$ such that

$$
V_{\eta}(x)=H_{\eta}\left(\varphi(x)^{2}, \sigma(x)\right) \quad \forall x \in \Sigma_{\beta_{0}} .
$$

We observe that the lifting is positive and satisfies

$$
R_{1-\eta}=1-R_{\eta}
$$

If $\eta \in C^{1}(\Sigma)$ then $R_{\eta} \in C^{0,1}(\bar{\Omega})$.

The first step in the proof of the theorem is the following integral estimate.

Lemma 2.3. Let $K$ and $u$ be as in the statement of the theorem. Assume that $\operatorname{diam} K<$ $\beta_{1}:=\beta_{0}(\Omega) / 4$ and let $x_{0}$ be a point on $\partial \Omega$ such that $K \subset B_{\beta_{1}}\left(x_{0}\right)$. Let $\eta \in C^{2}(\partial \Omega)$ be a function such that

$$
0 \leq \eta \leq 1, \quad \eta= \begin{cases}0 & \text { in a neighborhood } A_{\eta} \text { of } K, A_{\eta} \subset B_{\beta_{1}}\left(x_{0}\right), \\ 1 \quad \text { in } \partial \Omega \backslash B_{2 \beta_{1}}\left(x_{0}\right)\end{cases}
$$

Put $\zeta:=\varphi R_{\eta}^{2 q^{\prime}}$. Then

$$
\int_{\Omega}\left(u^{q} \varphi+u\right) R_{\eta}^{2 q^{\prime}} d x \leq c\|1-\eta\|_{W^{2 / q, q^{\prime}}(\Sigma)}^{q^{\prime}},
$$

where $c$ depends only on $q, N, \Omega$ and the choice of $\beta_{0}$.

Proof. If $F \in C(\Omega) \cap C^{2}\left(\Omega \backslash \Sigma_{\beta_{0}}\right)$ put

$$
\begin{aligned}
\tilde{\Delta} F & =\chi_{\Omega_{\beta_{0}}} \Delta F+\chi_{\Omega_{\beta_{0}}^{\prime}} \Delta F, \quad \tilde{\nabla} F=\chi_{\Omega_{\beta_{0}}} \nabla F+\chi_{\Omega_{\beta_{0}}^{\prime}} \nabla F, \\
F^{e} & =\chi_{\Omega_{\beta_{0}}} F, \quad F^{i}=\chi_{\Omega_{\beta_{0}}^{\prime}} F,
\end{aligned}
$$


where $\chi_{E}$ denotes the characteristic function of the set $E$. Note that $R_{\eta}^{e}$ (resp. $R_{\eta}^{i}$ ) can be extended as a function in $C^{1}\left(\bar{\Omega}_{\beta_{0}}\right)\left(\right.$ resp. $C^{1}\left(\bar{\Omega}_{\beta_{0}}^{\prime}\right)$ ).

We observe that

$$
\int_{\Omega_{\beta_{0}}}\left(-u \Delta \zeta+u^{q} \zeta\right) d x=\int_{\Sigma_{\beta_{0}}}\left(\zeta \frac{\partial u}{\partial \bar{v}}-u \frac{\partial \zeta^{e}}{\partial \bar{v}}\right) d S
$$

where $\bar{v}$ is the unit normal on $\Sigma_{\beta_{0}}$ pointing outward with respect to $\Omega_{\beta_{0}}$. This is verified in the same way as in [15, Thm. 3.3] (see proof of (3.11) there). In addition

$$
\int_{\Omega_{\beta_{0}}^{\prime}}\left(-u \Delta \zeta+u^{q} \zeta\right) d x=-\int_{\Sigma_{\beta_{0}}}\left(\zeta \frac{\partial u}{\partial \bar{v}}-u \frac{\partial \zeta^{i}}{\partial \bar{v}}\right) d S
$$

Hence

$$
\int_{\Omega}\left(-u \tilde{\Delta} \zeta+u^{q} \zeta\right) d x=-\int_{\Sigma_{\beta_{0}}} u\left(\frac{\partial \zeta^{e}}{\partial \bar{v}}-\frac{\partial \zeta^{i}}{\partial \bar{v}}\right) d S
$$

By a straightforward computation

$$
\begin{aligned}
\tilde{\Delta} \zeta= & -R_{\eta}^{2 q^{\prime}}+\varphi \tilde{\Delta} R_{\eta}^{2 q^{\prime}}+2 \nabla \varphi \cdot \tilde{\nabla} R_{\eta}^{2 q^{\prime}} \\
= & -R_{\eta}^{2 q^{\prime}}+2 q^{\prime} \varphi R_{\eta}^{2 q^{\prime}-1} \tilde{\Delta} R_{\eta}+2 q^{\prime}\left(2 q^{\prime}-1\right) \varphi R_{\eta}^{2 q^{\prime}-2}\left|\tilde{\nabla} R_{\eta}\right|^{2} \\
& +4 q^{\prime} R_{\eta}^{2 q^{\prime}-1} \nabla \varphi \cdot \tilde{\nabla} R_{\eta} .
\end{aligned}
$$

Therefore, by 2.14,

$$
\int_{\Omega}\left(R_{\eta}^{2 q^{\prime}} u+u^{q} \zeta\right) d x=2 q^{\prime} \int_{\Omega} u R_{\eta}^{2 q^{\prime}-2} G_{\eta} d x-\int_{\Sigma_{\beta_{0}}} u\left(\frac{\partial \zeta^{e}}{\partial \bar{v}}-\frac{\partial \zeta^{i}}{\partial \bar{v}}\right) d S
$$

where

$$
G_{\eta}=\varphi R_{\eta} \tilde{\Delta} R_{\eta}+\left(2 q^{\prime}-1\right) \varphi\left|\tilde{\nabla} R_{\eta}\right|^{2}+2 R_{\eta} \nabla \varphi \cdot \tilde{\nabla} R_{\eta}
$$

Further we obtain

$$
\frac{\partial \zeta^{e}}{\partial \bar{v}}=\frac{\partial \varphi}{\partial \bar{v}} R_{\eta}^{2 q^{\prime}-1}+2 q^{\prime} \varphi R_{\eta}^{2 q^{\prime}-1} \frac{\partial R_{\eta}^{e}}{\partial \bar{v}}, \quad \frac{\partial \zeta^{i}}{\partial \bar{v}}=\frac{\partial \varphi}{\partial \bar{v}} R_{\eta}^{2 q^{\prime}-1}+2 q^{\prime} \varphi R_{\eta}^{2 q^{\prime}-1} \frac{\partial R_{\eta}^{i}}{\partial \bar{v}},
$$

and hence

$$
\left|\frac{\partial \zeta^{e}}{\partial \bar{v}}-\frac{\partial \zeta^{i}}{\partial \bar{v}}\right| \leq 2 q^{\prime} \varphi R_{\eta}^{2 q^{\prime}-1}\left(\left|\frac{\partial R_{\eta}^{e}}{\partial \bar{v}}\right|+\left|\frac{\partial R_{\eta}^{i}}{\partial \bar{v}}\right|\right)
$$

Put

$$
\begin{aligned}
& M_{\eta}=\varphi^{1 / q^{\prime}}\left(\left|\tilde{\nabla} R_{\eta}\right|^{2}+\left|\tilde{\Delta} R_{\eta}\right|\right)+\varphi^{-1 / q}\left|\nabla \varphi \cdot \tilde{\nabla} R_{\eta}\right|, \\
& N_{\eta}=\varphi^{1 / q^{\prime}}\left(\left|\frac{\partial R_{\eta}^{e}}{\partial \bar{v}}\right|+\left|\frac{\partial R_{\eta}^{i}}{\partial \bar{v}}\right|\right) .
\end{aligned}
$$

Since $0<R_{\eta} \leq 1$ it follows that

$$
\left|G_{\eta}\right| \leq 2 q^{\prime} \varphi^{1 / q} M_{\eta}, \quad\left|\frac{\partial \zeta^{e}}{\partial \bar{v}}-\frac{\partial \zeta^{i}}{\partial \bar{v}}\right| \leq 2 q^{\prime} \zeta^{1 / q} N_{\eta} .
$$


Hence

$$
\begin{aligned}
\int_{\Omega} u R_{\eta}^{2 q^{\prime}-2}\left|G_{\eta}\right| d x & \leq 2 q^{\prime} \int_{\Omega} u \zeta^{1 / q} M_{\eta} d x \\
& \leq 2 q^{\prime}\left(\int_{\Omega} u^{q} \zeta d x\right)^{1 / q}\left(\int_{\Omega} M_{\eta}^{q^{\prime}} d x\right)^{1 / q^{\prime}}, \\
\int_{\Sigma_{\beta_{0}}} u\left|\frac{\partial \zeta^{e}}{\partial \bar{v}}-\frac{\partial \zeta^{i}}{\partial \bar{v}}\right| d S & \leq 2 q^{\prime} \int_{\Sigma_{\beta_{0}}} u \zeta^{1 / q} N_{\eta} d S \\
& \leq 2 q^{\prime}\left(\int_{\Sigma_{\beta_{0}}} u^{q} \zeta d S\right)^{1 / q}\left(\int_{\Sigma_{\beta_{0}}} N_{\eta}^{q^{\prime}} d S\right)^{1 / q^{\prime}} .
\end{aligned}
$$

Next we show that

$$
\int_{\Sigma_{\beta_{0}}} u^{q} \zeta d S \leq c \int_{\Omega}\left(R_{\eta}^{2 q^{\prime}} u+u^{q} \zeta\right) d x
$$

In view of the Keller-Osserman inequality

$$
\int_{\Sigma_{\beta_{0}}} u^{q} \zeta d S \leq c_{1}\left(\beta_{0}\right) \int_{\Sigma_{\beta_{0}}} u \zeta d S
$$

Let $\varphi^{\prime}$ be the solution of

$$
-\Delta \varphi^{\prime}=1 \quad \text { in } \Omega_{\beta_{0}}^{\prime}, \quad \varphi^{\prime}=0 \quad \text { on } \Sigma_{\beta_{0}}=\partial \Omega_{\beta_{0}}^{\prime} .
$$

Put

$$
c_{2}\left(\beta_{0}\right):=\max _{\Sigma_{\beta_{0}}}\left|\partial_{\mathbf{n}^{\prime}} \varphi^{\prime}\right|, \quad c_{3}\left(\beta_{0}\right):=\max _{\Omega_{\beta_{0}}^{\prime}} \varphi,
$$

where $\partial_{\mathbf{n}^{\prime}}$ denotes the derivative in the direction of the outward normal on $\Sigma_{\beta_{0}}=\partial \Omega_{\beta_{0}}^{\prime}$. Then

$$
\begin{aligned}
\int_{\Omega_{\beta_{0}}^{\prime}}\left(u+u^{q} \varphi^{\prime}\right) d x & =-\int_{\Sigma_{\beta_{0}}} u \partial_{\mathbf{n}^{\prime}} \varphi^{\prime} d S \\
& \geq c_{2}\left(\beta_{0}\right) \int_{\Sigma_{\beta_{0}}} u d S \geq \frac{c_{2}\left(\beta_{0}\right)}{c_{3}\left(\beta_{0}\right)} \int_{\Sigma_{\beta_{0}}} u \zeta d S .
\end{aligned}
$$

Let $\omega$ denote the characteristic function of $\Sigma \backslash B_{2 \beta_{1}}\left(x_{0}\right)$ and put

$$
c_{4}\left(\beta_{0}\right)=\inf _{\Omega_{\beta_{0}}^{\prime}} R_{\omega}^{2 q^{\prime}}
$$

By $2.10, \eta \geq \omega$; hence

$$
R_{\eta}^{2 q^{\prime}} \geq c_{4}\left(\beta_{0}\right) \quad \forall x \in \Omega_{\beta_{0}}^{\prime} .
$$

Using this inequality and the fact that $\varphi^{\prime}<\varphi$ we obtain

$$
\int_{\Omega_{\beta_{0}}^{\prime}}\left(u+u^{q} \varphi^{\prime}\right) d x \leq \frac{1}{c_{4}\left(\beta_{0}\right)} \int_{\Omega_{\beta_{0}}^{\prime}}\left(u+u^{q} \varphi\right) R_{\eta}^{2 q^{\prime}} d x .
$$


Inequalities (2.21)-2.24) imply 2.20). Further 2.15)-2.20) imply

$$
\int_{\Omega}\left(u+u^{q} \varphi\right) R_{\eta}^{2 q^{\prime}} d x \leq c\left(\int_{\Omega} M_{\eta}^{q^{\prime}} d x+\int_{\Sigma_{\beta_{0}}} N_{\eta}^{q^{\prime}} d S\right) .
$$

Put $\eta^{*}=1-\eta$ and note that

$$
M_{\eta}=M_{\eta^{*}}, \quad N_{\eta}=N_{\eta *} .
$$

We turn to the estimate of the terms on the right hand side of 2.25. By [15, Lemma 1.2],

$$
\left.\| \varphi^{1 / q^{\prime}}\left|\Delta R_{\eta^{*}}\right|\right)+\varphi^{-1 / q}\left|\nabla \varphi \cdot \nabla R_{\eta^{*}}\right|\left\|_{L^{q^{\prime}}\left(\Omega_{\beta_{0}}\right)} \leq c\right\| \eta^{*} \|_{W^{2 / q, q^{\prime}}(\Sigma)} .
$$

In order to estimate the norm of $\varphi^{1 / q^{\prime}}\left(\left|\nabla R_{\eta^{*}}\right|^{2}\right.$ in $L^{q^{\prime}}\left(\Omega_{\beta_{0}}\right)$ we proceed as in the proof of [15, Th. 3.3] (see (3.24) and the argument following it). First we obtain

$$
\begin{aligned}
\int_{\Omega_{\beta_{0}}} \varphi(\mid \nabla & \left.R_{\eta^{*}}\right|^{2 q^{\prime}} d x \\
& \leq c \int_{\Sigma} \int_{0}^{\rho_{0}}\left(\rho^{q^{\prime}}\left|\frac{\partial H_{\eta^{*}}(\rho, \sigma)}{\partial \rho}\right|^{2 q^{\prime}}+\left|\nabla_{\sigma} H_{\eta^{*}}(\rho, \sigma)\right|^{2 q^{\prime}}\right) d \rho d \sigma,
\end{aligned}
$$

where $\rho_{0}=\sup _{\sigma \in \Sigma} \varphi^{2}\left(\beta_{0}, \sigma\right)$, and then, estimating the terms on the right hand side by employing interpolation inequalities, we obtain

$$
\int_{\Omega_{\beta_{0}}} \varphi\left(\left|\tilde{\nabla} R_{\eta^{*}}\right|^{2 q^{\prime}} d x \leq c\left\|\eta^{*}\right\|_{W^{2 / q, q^{\prime}}(\Sigma)}^{q^{\prime}} .\right.
$$

Inequalities (2.26) and (2.28) yield

$$
\left\|M_{\eta^{*}}\right\|_{L^{q^{\prime}}\left(\Omega_{\beta_{0}}\right)} \leq c\left\|\eta^{*}\right\|_{W^{2 / q, q^{\prime}}(\Sigma)} .
$$

Next we estimate the norm of $M$ in $L^{q^{\prime}}\left(\Omega_{\beta_{0}}^{\prime}\right)$. Recall that, in $\Omega_{\beta_{0}}^{\prime}, R_{\eta^{*}}=V_{\eta^{*}}$ (see Definition 2.2p; since $V_{\eta^{*}}$ is harmonic,

$$
\begin{aligned}
M_{\eta^{*}} & =\varphi^{1 / q^{\prime}}\left|\nabla V_{\eta^{*}}\right|^{2}+\varphi^{-1 / q}\left|\nabla \varphi \cdot \nabla V_{\eta^{*}}\right|, \\
& \leq c\left(\left|\nabla V_{\eta^{*}}\right|^{2}+\left|\nabla V_{\eta^{*}}\right|\right) \quad \text { in } \Omega_{\beta_{0}}^{\prime} .
\end{aligned}
$$

Since $H_{\eta}$ is a solution of 2.7] it follows that, for every $\beta \in\left(0, \beta_{0}\right)$,

$$
\left\|H_{\eta^{*}}\right\|_{C^{2}\left(\left[\beta, \beta_{0}\right] \times \Sigma\right)} \leq c(\beta)\left\|\eta^{*}\right\|_{L^{q^{\prime}}(\Sigma)} .
$$

Let $v$ be the function given by

$$
v(x):=H_{\eta^{*}}\left(\varphi(x)^{2}, \sigma(x)\right) \quad \forall x \in \bar{\Omega}_{\beta_{0}} .
$$

Then

$$
\|v\|_{C^{2}\left(\Omega_{\beta_{0}} \backslash \Omega_{\beta}\right)} \leq c^{\prime}(\beta)\left\|\eta^{*}\right\|_{L^{q^{\prime}(\Sigma)}} .
$$


Since $V_{\eta^{*}}$ is the Poisson potential of $\left.v\right|_{\Sigma_{\beta_{0}}}$ in $\Omega_{\beta_{0}}^{\prime}$, it follows that

$$
\left\|V_{\eta^{*}}\right\|_{C^{1}\left(\bar{\Omega}_{\beta_{0}}^{\prime}\right)} \leq c^{\prime}\left(\beta_{0}\right)\left\|\eta^{*}\right\|_{L^{q^{\prime}}(\Sigma)} .
$$

Therefore, in view of 2.30 and 2.10,

$$
\left\|M_{\eta^{*}}\right\|_{L^{q^{\prime}\left(\Omega_{\beta_{0}}^{\prime}\right)}} \leq c\left\|\eta^{*}\right\|_{L^{q^{\prime}(\Sigma)}} .
$$

By 2.29) and 2.33,

$$
\left\|M_{\eta^{*}}\right\|_{L^{q^{\prime}(\Omega)}} \leq c\left\|\eta^{*}\right\|_{W^{2 / q, q^{\prime}(\Sigma)}} .
$$

Since $v=R_{\eta^{*}}^{e}, V_{\eta^{*}}=R_{\eta^{*}}^{i}, 2.31$ and 2.32 imply

$$
\left\|N_{\eta^{*}}\right\|_{L^{q^{\prime}}\left(\Sigma_{\beta_{0}}\right)} \leq c\left\|\eta^{*}\right\|_{L^{q^{\prime}}(\Sigma)} .
$$

Finally, 2.34, 2.35) and 2.25) imply 2.11.

At this point we need some additional notations. If $V$ is an open set in $\mathbb{R}^{d}$ we denote by $C_{\alpha, p}^{V}(K)$ the $C_{\alpha, p}$-capacity of a compact set $K \subset V$ relative to $V$, i.e., relative to test functions with compact support in $V$. It is known that

$$
C_{\alpha, p}^{V}(K)=r^{d-\alpha p} C_{\alpha, p}^{V / r}(K / r) \quad \forall K \subset V, K \text { compact. }
$$

Furthermore, for every positive $\theta$, there exists a constant $c_{\theta}$ such that, for every compact set $K \subset V$ which satisfies $\operatorname{dist}(K, \partial V)>\theta$,

$$
C_{\alpha, p}(K) \leq C_{\alpha, p}^{V}(K) \leq c_{\theta} C_{\alpha, p}(K) .
$$

If $V$ is a relatively open subset of $\Sigma, F$ is a compact subset of $V$ and $C_{\alpha, p}$ denotes capacity on $\Sigma$ then

$$
C_{\alpha, p}^{V}(K) \approx r^{N-1-\alpha p} C_{\alpha, p}^{V / r}(K / r) \quad \forall K \subset F, K \text { compact, }
$$

and 2.37 remains valid. Finally, for every $x_{0} \in \Sigma$, we define

$$
\Gamma_{s}\left(x_{0}\right):=B_{s}\left(x_{0}\right) \cap \Sigma, \quad D_{s}\left(x_{0}\right):=\Omega \backslash B_{s}\left(x_{0}\right) .
$$

Lemma 2.4. Let $K$ and $u$ be as in the statement of the theorem. Assume that diam $K<$ $\gamma<\beta_{1}$ and let $x_{0} \in \Sigma$ be a point such that $K \subset \Gamma_{\gamma}\left(x_{0}\right)$. Then there exists a constant $c$, depending only on $q, N, \Omega$ and the choice of $\beta_{0}$, such that

$$
\int_{D_{3 \gamma}\left(x_{0}\right)}\left(u^{q} \varphi+u\right) d x \leq c \gamma^{N-1-2 /(q-1)} C_{2 / q, q^{\prime}}(K / \gamma)
$$

where $\varphi$ is as in Definition 2.2

Proof. Let $\eta \in C^{2}(\partial \Omega)$ be a function such that

$$
0 \leq \eta \leq 1, \quad \eta= \begin{cases}0 & \text { in a neighborhood } A_{\eta} \text { of } K, A_{\eta} \subset B_{\gamma}\left(x_{0}\right), \\ 1 & \text { in } \partial \Omega \backslash B_{2 \gamma}\left(x_{0}\right) .\end{cases}
$$

By Lemma 2.3 inequality 2.11 holds. 
If $\omega_{\gamma}$ denotes the characteristic function of $\Sigma \backslash \Gamma_{2 \gamma}$ then

$$
R_{\eta} \geq R_{\omega_{\gamma}} \text {. }
$$

In addition there exists $c_{0}>0$, depending only on $\Omega$ and $\beta_{0}$, such that

$$
\inf _{D_{3 \gamma}\left(x_{0}\right)} R_{\omega_{\gamma}}>c_{0}
$$

for every $\gamma \in\left(0, \beta_{0} / 4\right)$ and every $x_{0} \in \Sigma$. This follows in a straightforward manner from Definition 2.2. Consequently, if $\eta$ satisfies 2.41,

$$
R_{\eta} \geq c \quad \text { in } D_{3 \gamma}\left(x_{0}\right) .
$$

This fact and 2.11) imply that

$$
\int_{\Omega \backslash B_{3 \gamma}\left(x_{0}\right)}\left(u^{q} \varphi+u\right) d x \leq c\|1-\eta\|_{W^{2 / q, q^{\prime}}(\Sigma)}^{q^{\prime}} .
$$

Taking the infimum over all such functions $\eta$ we obtain

$$
\int_{D_{3 \gamma}\left(x_{0}\right)}\left(u^{q} \varphi+u\right) d x \leq c C_{2 / q, q^{\prime}}^{\Gamma_{2 \gamma}\left(x_{0}\right)}(K) .
$$

By 2.37) and 2.38,

$$
C_{2 / q, q^{\prime}}^{\Gamma_{2 \gamma}\left(x_{0}\right)}(K) \approx \gamma^{N-1-2 /(q-1)} C_{2 / q, q^{\prime}}^{\Gamma_{2}\left(x_{0}\right)}(K / \gamma) \leq c \gamma^{N-1-2 /(q-1)} C_{2 / q, q^{\prime}}(K / \gamma) .
$$

The last two inequalities imply 2.40$]$.

Lemma 2.5. Let $K$ and $u$ be as in the statement of the theorem. Pick $\gamma$ such that diam $K$ $<\gamma<\beta_{0} / 16$. Let $x_{0} \in \Sigma$ be a point such that $K \subset \Gamma_{3 \gamma / 2}\left(x_{0}\right)$. Then there exists $a$ constant $c$, depending only on $q, N, \Omega$ and the choice of $\beta_{0}$, such that

$$
\int_{\Omega \cap \partial B_{4 \gamma}\left(x_{0}\right)} u \rho d S \leq c \gamma^{N-2 /(q-1)} C_{2 / q, q^{\prime}}(K / \gamma) .
$$

Proof. Put

and

$$
h_{\tau}\left(x ; x_{0}\right)= \begin{cases}\frac{1}{N-2}\left(1-\frac{\tau^{N-2}}{\left|x-x_{0}\right|^{N-2}}\right) & \text { if } N \geq 3, \\ 1-\frac{\tau}{\left|x-x_{0}\right|} & \text { if } N=2,\end{cases}
$$

$$
\psi_{\tau}\left(x ; x_{0}\right)=\phi(x) h_{\tau}\left(x ; x_{0}\right) \quad \forall x \in \Omega,
$$

where $d(\Omega)=\operatorname{diam} \Omega$ and $\phi$ is the eigenfunction corresponding to the first eigenvalue of $-\Delta$ in $\Omega$, normalized so that $\max \phi=1$. Assuming $\tau \leq \beta_{0} / 4$, we get

$$
\int_{D_{\tau}\left(x_{0}\right)} u^{q} \psi_{\tau} d x=\int_{D_{\tau}\left(x_{0}\right)} u \Delta \psi_{\tau} d x-\int_{\Omega \cap \partial B_{\tau}\left(x_{0}\right)} u \partial_{\mathbf{n}} \psi_{\tau} d S
$$

where $\partial_{\mathbf{n}}$ denotes the directional derivative in the direction of the outward unit normal on $D_{\tau}\left(x_{0}\right)$. Note that

$$
-\partial_{\mathbf{n}} \psi_{\tau}(x)=\phi(x) / \tau \geq c \rho(x) / \tau \quad \forall x \in \Omega \cap \partial B_{\tau}\left(x_{0}\right) .
$$




$$
\Delta \psi_{\tau}= \begin{cases}-\lambda_{1} \phi h_{\tau}+2 \frac{\tau^{N-2}\left\langle x-x_{0}, \nabla \phi(x)\right\rangle}{\left|x-x_{0}\right|^{N}} & \text { if } N>2, \\ -\lambda_{1} \phi h_{\tau}-2 \frac{\tau \phi}{\left|x-x_{0}\right|^{3}}+2 \frac{\tau\left\langle x-x_{0}, \nabla \phi(x)\right\rangle}{\left|x-x_{0}\right|^{2}} & \text { if } N=2 .\end{cases}
$$

We claim that there exists a constant $b(\Omega)>0$ such that

$$
\inf _{\substack{x \in \Omega \\ x_{0} \in \Sigma}} \frac{\left\langle x-x_{0}, \nabla \phi\right\rangle}{\left|x-x_{0}\right|^{2}} \geq-b(\Omega) .
$$

Since $\left|\nabla \phi(x)-\nabla \phi\left(x_{0}\right)\right| /\left|x-x_{0}\right|$ is bounded it is sufficient to verify that there exists a constant $c$ such that

$$
I\left(x, x_{0}\right):=\frac{\left\langle x-x_{0}, \nabla \phi\left(x_{0}\right)\right\rangle}{\left|x-x_{0}\right|^{2}}>-c
$$

for all $x_{0} \in \Sigma$ and $x \in \Omega$. Put $R=\beta_{0} / 2$ and

$$
\left|x-x_{0}\right|=r, \quad \bar{x}_{0}=x_{0}+v\left(x_{0}\right) R, \quad z=\cos \left(\frac{x-x_{0}}{\left|x-x_{0}\right|}, v\left(x_{0}\right)\right) .
$$

(Recall that $v\left(x_{0}\right)$ denotes the outward unit normal on $\partial \Omega$ at $x_{0}$.) Then the ball $B_{R}\left(\bar{x}_{0}\right)$ lies outside $\Omega$ and touches $\partial \Omega$ only at the point $x_{0}$.

For every $x \in \Omega$,

$$
R^{2} \leq\left|x-\bar{x}_{0}\right|^{2}=r^{2}+R^{2}-2 r R z .
$$

Hence $z \leq r / 2 R$. Since $\nabla \phi\left(x_{0}\right)=-\left|\nabla \phi\left(x_{0}\right)\right| v\left(x_{0}\right)$ this implies 2.52) with

$$
c=\frac{1}{\beta_{0}} \inf _{\Sigma}\left|\nabla \phi\left(x_{0}\right)\right| .
$$

Thus (2.51) is verified. Using 2.52) we also obtain, for $x \in D_{\tau}\left(x_{0}\right)$,

$$
\frac{\tau \phi(x)}{\left|x-x_{0}\right|^{3}}=\frac{\tau \nabla \phi\left(x_{0}\right) \cdot\left(x-x_{0}\right)}{\left|x-x_{0}\right|^{3}}+O(1) \geq I\left(x, x_{0}\right)+O(1) \geq-c_{1},
$$

where $c_{1}$ is a constant depending only on $N$ and $\Omega$.

Note that $\sup _{D_{\tau}\left(x_{0}\right)} h_{\tau}\left(\cdot ; x_{0}\right)$ is bounded by a constant independent of $\tau$ or $x_{0}$. (Recall that $0<\tau<\beta_{0} / 4$.) Therefore, 2.50, 2.51) and 2.53) imply

$$
\Delta \psi_{\tau} \geq-A \text { in } D_{\tau}\left(x_{0}\right),
$$

where $A$ is a constant depending only on $N$ and $\Omega$. By (2.48), (2.49) and 2.54),

$$
\int_{D_{\tau}\left(x_{0}\right)}\left(u^{q} \phi+u\right) d x \geq \frac{A}{\tau} \int_{\Omega \cap \partial B_{\tau}\left(x_{0}\right)} u \rho d S
$$

for every $\tau \in\left(0, \beta_{0} / 4\right)$ and $x_{0} \in \Sigma$. Finally, (2.40) and (2.55) imply (2.45).

Remark. The constant in inequality 2.45 is independent of $\gamma$ but depends on $\Omega$. However the inequality is invariant with respect to dilation: if $\Omega$ and $K$ are replaced by 
$\Omega^{\kappa}:=\kappa\left(\Omega-x_{0}\right)$ and $K^{\kappa}=\kappa\left(K-x_{0}\right)$ respectively, then 2.45 remains valid with the same constant $c$. To simplify the notation assume that $x_{0}=0$. If $U$ is a solution of our equation in $\Omega^{\kappa}$ which vanishes on $\partial \Omega^{\kappa} \backslash K^{\kappa}$ then $u(x)=\kappa^{2 /(q-1)} U(\kappa x)$ is a solution in $\Omega$ which vanishes on $\partial \Omega \backslash K$. Therefore 2.45 holds with respect to $u$ and we obtain

$$
\kappa^{2 /(q-1)} \int_{\Omega \cap \partial B_{3 \gamma}(0)} U(\kappa x) \rho(x) d S(x) \leq c \gamma^{N-2 /(q-1)} C_{2 / q, q^{\prime}}(K / \gamma) .
$$

Put $\xi:=\kappa x$. Then $\rho_{\partial \Omega^{\kappa}}(\xi)=\kappa \rho(x)$ and we obtain

$$
\int_{\Omega^{\kappa} \cap \partial B_{3 \kappa \gamma}(0)} U(\xi) \rho_{\partial \Omega^{\kappa}}(\xi) d S(\xi) \leq c(\kappa \gamma)^{N-2 /(q-1)} C_{2 / q, q^{\prime}}\left(K^{\kappa} /(\kappa \gamma)\right) .
$$

Lemma 2.6. Let $K, u$ be as in the statement of the theorem. Then there exists $\gamma_{0}=$ $\gamma_{0}(\Omega)<10^{-3} \beta_{0}$ such that the following statement holds.

Assume that $\operatorname{diam}(K)<\gamma<\gamma_{0}$. Then there exists a constant $c$, depending only on $q, N, \Omega$ and the choice of $\beta_{0}$, such that

$$
\int_{D_{9 \gamma / 2,5 \gamma}\left(x_{0}\right)} u \rho d x \leq c \gamma^{1+N-2 /(q-1)} C_{2 / q, q^{\prime}}(K / \gamma)
$$

for every $\delta \in[0, \gamma / 4)$ and $x_{0} \in \Sigma_{\delta}$ such that $K \subset \Gamma_{5 \gamma / 4}\left(x_{0}\right)$. Here

$$
D_{r, r^{\prime}}\left(x_{0}\right):=\left\{x \in \Omega: r<\left|x-x_{0}\right|<r^{\prime}\right\} .
$$

Proof. If $z=\left(z_{1}, 0, \ldots, 0\right)$ with $0 \leq z_{1}<1$ then

$$
\left\{x \in \mathbb{R}_{+}^{N}: 17 / 4<|x-z|<5\right\} \subset\left\{x \in \mathbb{R}_{+}^{N}: 4<|x|<6\right\} .
$$

Therefore, as $\Omega$ is bounded of class $C^{2}$,

$$
\left\{x \in \Omega: 9 \gamma / 2<\left|x-x_{0}\right|<5 \gamma\right\} \subset\left\{x \in \Omega: 4 \gamma<\left|x-\sigma\left(x_{0}\right)\right|<7 \gamma\right\}
$$

for every $x_{0} \in \Sigma_{\delta}$, provided that $0 \leq \delta<\gamma<\gamma_{0}$ and $\gamma_{0}$ is sufficiently small. The value of $\gamma_{0}$ depends only on the $C^{2}$ "norm" of $\partial \Omega$.

By Lemma 2.5 if $K \subset \Gamma_{3 \gamma / 2}\left(\sigma\left(x_{0}\right)\right)$,

$$
\int_{\Omega \cap \partial B_{4 \gamma}\left(\sigma\left(x_{0}\right)\right)} u \rho d S \leq c \gamma^{N-2 /(q-1)} C_{2 / q, q^{\prime}}(K / \gamma) .
$$

The assumptions of the present lemma imply that the above condition on $K$ is satisfied. Hence,

$$
\int_{\Omega \cap \partial B_{t \gamma}\left(\sigma\left(x_{0}\right)\right)} u \rho d S \leq c \gamma^{N-2 /(q-1)} C_{2 / q, q^{\prime}}(K / \gamma)
$$

for every $t \in[4,7]$, with $c$ independent of $t$. Integrating over $t$ we obtain

$$
\int_{D_{4 \gamma, 7 \gamma}\left(\sigma\left(x_{0}\right)\right)} u \rho d x \leq c \gamma^{1+N-2 /(q-1)} C_{2 / q, q^{\prime}}(K / \gamma) .
$$

This inequality and 2.57) imply 2.56. 
Proof of Theorem 2.1. Let $\gamma_{0}(\Omega)$ and $m(\Omega)$ be as in Lemma 2.6 and Theorem C.1 respectively. Assume that $\operatorname{diam} K<\theta:=\min \left(\gamma_{0}, \beta_{0} / 40 m\right)$ and pick $\gamma \in(\operatorname{diam} K, \theta)$. Let $x_{0} \in \Omega$ be a point such that

$$
\rho_{\partial \Omega}\left(x_{0}\right)=\rho_{K}\left(x_{0}\right)=\delta:=\gamma / 4 \text {. }
$$

Then $K \subset B_{5 \gamma / 4}\left(x_{0}\right)$ and Lemma 2.6 applies, so that inequality 2.56 holds.

Denote by $\mathcal{P}_{\tau}^{x_{0}}$ the Poisson kernel of $D_{\tau}\left(x_{0}\right)$. Since $u$ is subharmonic,

$$
u(x) \leq v(x):=\int_{y \in \Omega:\left|y-x_{0}\right|=\tau} P_{\tau}(x, y) u(y) d S(y)
$$

for every $\tau>5 \gamma / 4$. Assume, as we may, that $m(\Omega)>20$. Then

$$
\tau \in[9 \gamma / 2,5 \gamma] \Longrightarrow \tau<\min \left(\beta_{0} / 8 m, m \delta\right) .
$$

Assuming that $\tau$ satisfies this condition, it follows, by Theorem C.1 that there exists a constant $c$, independent of $\tau$ and $x_{0}$, such that

$$
\mathcal{P}_{\tau}^{x_{0}}(x, y) \leq c \frac{\rho(x) \rho(y)}{\tau|x-y|^{N}}
$$

in the set

$$
E_{\Omega}\left(\tau, x_{0}\right)=\left\{(x, y) \in \Omega \times \Omega:\left|y-x_{0}\right|=\tau,\left|x-x_{0}\right| \geq 4 \tau\right\} .
$$

Note that, in this set, $|x-y| \approx\left|x-x_{0}\right|$. Therefore, if $\left|x-x_{0}\right| \geq 4 \tau$,

$$
u(x) \leq c \frac{\rho(x)}{\tau\left|x-x_{0}\right|^{N}} \int_{y \in \Omega:\left|y-x_{0}\right|=\tau} \rho(y) u(y) d S(y) .
$$

This inequality holds for every $\tau \in[9 \gamma / 2,5 \gamma]$ and $x \in D_{4 \tau}\left(x_{0}\right)$. Therefore, integrating over $\tau$ in this interval yields

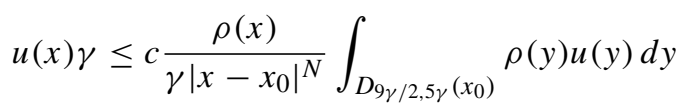

for every $x \in D_{20 \gamma}\left(x_{0}\right), \gamma \in(\operatorname{diam} K, \theta)$ and $x_{0}$ satisfying 2.60). Hence, by 2.56,

$$
u(x) \gamma \leq c \frac{\rho(x)}{\gamma\left|x-x_{0}\right|^{N}} \gamma^{1+N-2 /(q-1)} C_{2 / q, q^{\prime}}(K / \gamma)
$$

and consequently

$$
u(x) \leq c \rho(x) \gamma^{-1-2 /(q-1)} C_{2 / q, q^{\prime}}(K / \gamma),
$$

for every $x \in \Omega$ and $\gamma \in(\operatorname{diam} K, \theta)$ such that $\rho_{K}(x)>25 \gamma$. This inequality and Lemma B.2 imply that

$$
u(\xi) \leq c C_{2 / q, q^{\prime}}(K / \theta) \leq c C_{2 / q, q^{\prime}}\left(K / \beta_{0}\right) \quad \forall \xi \in \Omega: \rho_{K}(\xi)=\beta_{0} .
$$

By the maximum principle, applied in $D_{\beta_{0}}^{K}=\left\{x \in \Omega: \rho_{K}(x)>\beta_{0}\right\}$,

$$
u(\xi) \leq c C_{2 / q, q^{\prime}}\left(K / \beta_{0}\right) \quad \forall \xi \in D_{\beta_{0}}^{K},
$$


and by Lemma B.2,

$$
C_{2 / q, q^{\prime}}\left(K / \beta_{0}\right) \leq c C_{2 / q, q^{\prime}}(K / d(\Omega)) \leq c C_{2 / q, q^{\prime}}(K / \alpha) \quad \forall \alpha \in(0, d(\Omega)),
$$

where $d(\Omega):=\operatorname{diam} \Omega$. Consequently, 2.6) holds for every $\xi \in D_{\beta_{0}}^{K}$.

Next we show that, for every compact set $K \subset \Sigma$ and every integer $n>d(\Omega) / 20 \theta$, 2.6. holds (with a constant depending on $n$ ) for all points $\xi \in \Omega$ such that

$$
\frac{\operatorname{diam} \Omega}{n} \operatorname{diam} K \leq \rho_{K}(\xi) \text {. }
$$

There is a number $M_{n}$ such that $\partial \Omega$ can be covered by $M_{n}$ balls of diameter $\frac{\text { diam } \Omega}{20 n} \operatorname{diam} K$. Consequently, $K=\bigcup_{m=1}^{M_{n}} K_{m}$, where $K_{m}$ is compact and

$$
\operatorname{diam} K_{m}<\theta, \quad m=1, \ldots, M_{n} .
$$

Furthermore, if $\xi$ satisfies 2.69,

$$
20 \operatorname{diam} K_{m} \leq \rho_{K_{m}}(\xi), \quad m=1, \ldots, M_{n} .
$$

Hence

$$
U_{K_{m}}(\xi) \leq c \rho(\xi) \rho_{K_{m}}(\xi)^{-1-2 /(q-1)} C_{2 / q, q^{\prime}}\left(K_{m} / \rho_{K_{m}}(\xi)\right), \quad m=1, \ldots, M_{n} .
$$

However $u \leq \sum_{m=1}^{M_{n}} U_{K_{m}}$ and $\rho_{K}(\xi) \leq \rho_{K_{m}}(\xi)$. Therefore

$$
u(\xi) \leq c M_{n} \rho(x) \rho_{K}(\xi)^{-1-2 /(q-1)} C_{2 / q, q^{\prime}}\left(K / \rho_{K}(\xi)\right) .
$$

Thus 2.6 holds, with a constant $c$ depending on $n$, for every $\xi \in \Omega$ satisfying 2.69].

Finally we show that 2.6 holds for every $\xi \in \Omega$ such that

$$
\frac{1}{4} \operatorname{diam} K \geq \rho_{K}(\xi) \text {. }
$$

Let $\xi$ be such a point and put $\delta=\frac{1}{2} \rho_{K}(\xi)$. Let

$$
F_{j}(\xi):=\left\{x \in K: 2^{j-1} \delta \leq|x-\xi| \leq 2^{j} \delta\right\} .
$$

Let $J(\xi)=\max \left\{j \in \mathbb{N}: C_{2 / q, q^{\prime}}\left(F_{j}\right) \neq 0\right\}$. Then

$$
K=\bigcup_{j=1}^{J} F_{j}(\xi), \quad \operatorname{diam} F_{j} \leq 2^{j+1} \delta \leq 4 \rho_{F_{j}}(\xi) .
$$

Consequently,

$$
U_{F_{j}}(\xi) \leq c \rho(\xi) \rho_{F_{j}}(\xi)^{-1-2 /(q-1)} C_{2 / q, q^{\prime}}\left(F_{j} / \rho_{F_{j}}(\xi)\right),
$$

with $c$ independent of $j$. By [1] (see Remark below), taking into account the fact that $\rho_{F_{j}}(\xi) \geq 2^{j-1} \delta$, it follows that

$$
C_{2 / q, q^{\prime}}\left(F_{j} / \rho_{F_{j}}(\xi)\right) \leq C_{2 / q, q^{\prime}}\left(F_{j} / 2^{j-1} \delta\right) \leq c 2^{-(j-1)(N-1-2 /(q-1))} C_{2 / q, q^{\prime}}\left(F_{j} / \delta\right) .
$$


Therefore, by (2.71],

$$
\begin{aligned}
u(\xi) & \leq \sum_{j=1}^{J} U_{F_{j}}(\xi) \\
& \leq c \rho(\xi) \sum_{j=1}^{J} 2^{-(j-1)(1+2 /(q-1))} \delta^{-1-2 /(q-1)} 2^{-(j-1)(N-1-2 /(q-1))} C_{2 / q, q^{\prime}}(K / \delta) \\
& \leq c \rho(\xi) \delta^{-1-2 /(q-1)} C_{2 / q, q^{\prime}}(K / \delta) \sum_{j=1}^{\infty} 2^{-(j-1) N} \\
& \leq c \rho(\xi) \rho_{K}(\xi)^{-1-2 /(q-1)} C_{2 / q, q^{\prime}}\left(K / \rho_{K}(\xi)\right) .
\end{aligned}
$$

Remark. By the argument employed in the first part of the proof of [1, Thm. 5.2.1] (see in particular inequality (5.2.3)),

$$
C_{2 / q, q^{\prime}}(t E) \leq A t^{N-1-2 /(q-1)} C_{2 / q, q^{\prime}}(E) \quad \forall t \in(0,1],
$$

for every $q \geq q_{c}=(N+1) /(N-1)$ and every compact set $E \subset \partial \Omega$, with the constant $A$ depending only on $q, N, \partial \Omega$. (Recall that, for $q \geq q_{c}, 2 q^{\prime} / q=2 /(q-1) \leq N-1$.) In addition, for every positive number $\tau$,

$$
C_{2 / q, q^{\prime}}(\alpha E) \leq A C_{2 / q, q^{\prime}}(E) \quad \forall \alpha \in(0, \tau],
$$

where $A$ depends only on $q, N, \partial \Omega$ and $\tau$. For the proof of this assertion see Lemma B.2.

\section{Boundary singularities: estimates from below}

Given a point $\xi \in \mathbb{R}^{N}$, a set $K \subset \mathbb{R}^{N}$ and an integer $m$, put

$$
\begin{aligned}
S_{m}(\xi) & :=\left\{x \in \mathbb{R}^{N}: r_{m+1} \leq|x-\xi| \leq r_{m}\right\}, \quad r_{m}=2^{-m}, \\
K_{m}(\xi) & :=K \cap S_{m}(\xi) .
\end{aligned}
$$

In addition we define

$$
u_{K}=\sup \left\{u_{\mu}: \mu \in W_{+}^{2 / q, q^{\prime}}(\Sigma), \mu(\Sigma \backslash K)=0\right\} .
$$

Theorem 3.1. Assume that $q \geq q_{c}=(N+1) /(N-1)$ and let $K$ be a compact subset of $\partial \Omega$. Then there exists a constant $c=c(\Omega, N, q)$, independent of $K$, such that

$$
u_{K}(\xi) \geq c \rho(\xi) \sum_{m=-\ell(\Omega)}^{\infty} r_{m}^{-1-2 /(q-1)} C_{2 / q, q^{\prime}}\left(K_{m}(\xi) / r_{m}\right)
$$

for every point $\xi \in \Omega$. Here $\ell(\Omega)$ is an integer such that

$$
\operatorname{diam} \Omega \leq 2^{\ell(\Omega)}<2 \operatorname{diam} \Omega .
$$

Proof. Inequality (3.3) is invariant with respect to dilations. Therefore, without loss of generality, we assume that $\beta_{0}=1$. 
It is sufficient to prove 3.3 under the following additional conditions:

$$
\text { (i) } \operatorname{diam} K \leq 1 / 8, \quad \text { (ii) } \rho_{K}(\xi) \leq 1 / 8 \text {. }
$$

We verify this assertion in two steps.

First, assuming that 3.3 holds for $K$ and $\xi$ satisfying (3.4), we show that it holds for all $\xi \in \Omega$. Put

$$
\Omega_{\tau}^{K}=\left\{x \in \Omega: \rho_{K}(x)>\tau\right\} .
$$

In $\Omega_{1 / 8}^{K}$ inequality 3.3 is equivalent to

$$
u_{K}(\xi) \geq c \rho(\xi) C_{2 / q, q^{\prime}}(K)
$$

Clearly, for $\xi \in \Omega_{1 / 8}^{K}$, the right hand side of 3.3 is bounded above by $c \rho(\xi) C_{2 / q, q^{\prime}}(K)$ so that 3.6 implies 3.3 . On the other hand, 3.3 implies that for every $\xi \in \Omega_{1 / 8}^{K}$,

$$
\begin{aligned}
u_{K}(\xi) & \geq c \rho(\xi) \sum_{m=-\ell(\Omega)}^{3} r_{m}^{-1-2 /(q-1)} C_{2 / q, q^{\prime}}\left(K_{m}(\xi) / r_{m}\right) \\
& \geq c \rho(\xi) \sum_{m=-\ell(\Omega)}^{3} C_{2 / q, q^{\prime}}\left(K_{m}(\xi) / r_{m}\right) \geq c \rho(\xi) C_{2 / q, q^{\prime}}(K)
\end{aligned}
$$

By Theorem 2.1.

$$
u_{K}(\xi) \leq c(\tau) \rho(\xi) C_{2 / q, q^{\prime}}(K) \quad \forall \xi \in D_{\tau}^{K}
$$

Therefore, setting $\eta=u_{K}^{q-1}$, we have

$$
-\Delta u_{K}+\eta u_{K}=0 \quad \text { in } \Omega_{1 / 16}^{K},
$$

where $\sup _{\Omega_{1 / 16}^{K}}|\eta| \leq c$. By Hopf's lemma, $-\partial_{\mathbf{n}} u_{K}(x)>0$ for every $x \in \partial \Omega$ such that $\rho_{K}(x)>1 / 16$. Therefore $\inf _{\Omega \cap \Omega_{1 / 8}^{K}} u_{K} / \rho>0$. By (3.7) and our assumption,

$$
u_{K}(\xi) \approx \rho(\xi) C_{2 / q, q^{\prime}}(K) \quad \text { on } \Omega \cap \partial \Omega_{1 / 8}^{K}
$$

Consequently, there exists a constant $c$ depending only on $\Omega, q$ such that 3.6 holds in $\Omega_{1 / 8}^{K}$.

Next we show that if 3.3 holds for $K$ such that diam $K \leq 1 / 8$ then it holds for every compact set $K \subset \partial \Omega$. There exists a natural number $n_{0}$ such that $\Sigma$ can be covered by $n_{0}$ balls of diameter $1 / 16$. Therefore every compact set $K$ can be written as a union $\bigcup_{i=1}^{n_{0}} F_{i}$ of compact sets, each of which has diameter smaller than $1 / 8$. Since

$$
u_{K} \geq \frac{1}{n_{0}} \sum_{i=1}^{n_{0}} u_{F_{i}}
$$


we conclude that

$$
\begin{aligned}
u_{K}(\xi) & \geq \frac{c}{n_{0}} \rho(\xi) \sum_{i=1}^{n_{0}} \sum_{m=-\ell(\Omega)}^{\infty} r_{m}^{-1-2 /(q-1)} C_{2 / q, q^{\prime}}\left(\left(F_{i}\right)_{m}(\xi) / r_{m}\right) \\
& \geq c \rho(\xi) \sum_{m=-\ell(\Omega)}^{\infty} r_{m}^{-1-2 /(q-1)} C_{2 / q, q^{\prime}}\left(K_{m}(\xi) / r_{m}\right)
\end{aligned}
$$

for every $\xi \in \Omega$.

Assume that diam $K \leq 1 / 8$ and put

$$
A_{K}:=\left\{x \in \Omega: \rho_{K}(x) \leq 1 / 8\right\} .
$$

Then

$$
\operatorname{diam} A_{K} \leq 3 / 8, \quad K \subset B_{1 / 4}(\xi), \quad A_{K} \subset B_{3 / 8}(\xi) \quad \forall \xi \in A_{K} .
$$

Pick a point $x_{0} \in A_{K}$ and let $Q=Q\left(x_{0}\right)$ be a subdomain of $\Omega$ of class $C^{2}$ such that

$$
\Omega \cap \bar{B}_{3 / 8}\left(x_{0}\right) \subset Q\left(x_{0}\right) \subset \Omega \cap B_{1 / 2}\left(x_{0}\right) .
$$

By 3.11,

$$
A_{K} \subset Q\left(x_{0}\right) \subset B_{7 / 8}(\xi) \quad \forall \xi \in A_{K} .
$$

Let $u_{K}^{0}$ be defined as in (3.2) with $\Omega$ replaced by $Q\left(x_{0}\right)$. Then

$$
u_{K}^{0} \leq u_{K}, \quad \rho_{\partial Q}(x)=\rho_{\partial \Omega}(x) \quad \forall x \in A_{K} .
$$

The first assertion is obvious and the second is easily verified. Indeed, if it does not hold, there exists $x^{\prime} \in A_{K}$ such that $\rho_{\partial Q}\left(x^{\prime}\right)<\rho_{\partial \Omega}\left(x^{\prime}\right)$. Let $y^{\prime} \in \partial Q$ be a point such that $\rho_{\partial Q}\left(x^{\prime}\right)=\left|x^{\prime}-y^{\prime}\right|$. Then $y^{\prime} \notin \partial \Omega$ and therefore $\left|y^{\prime}-x_{0}\right| \geq 1 / 2$. On the other hand, by [3.11, $\left|x^{\prime}-x_{0}\right| \leq 3 / 8$, so that

$$
\rho_{\partial Q}\left(x^{\prime}\right) \geq\left|y^{\prime}-x_{0}\right|-\left|x^{\prime}-x_{0}\right| \geq 1 / 8 .
$$

This is impossible because $\rho_{\partial \Omega}\left(x^{\prime}\right) \leq 1 / 8$.

In the remaining part of the proof we establish the following:

Assertion 1. For every compact set $K \subset \partial \Omega$ and $x_{0} \in A_{K}$, if

$$
\operatorname{diam} K<1 / 8 \text {, }
$$

then inequality (3.3) is valid in $Q\left(x_{0}\right)$ (as previously defined), with $u_{K}$ replaced by $u_{K}^{0}$ and with a constant independent of $K$ and $Q\left(x_{0}\right)$.

First we verify that the assertion implies the statement of the theorem. Assumption (3.14) implies that $K \subset B_{1 / 4}\left(x_{0}\right)$ and $A_{K} \subset Q$. In view of (3.13), the assertion implies that (3.3) holds in $A_{K}$ for every compact set $K \subset \partial \Omega$ satisfying (3.14). Therefore, by the first part of the proof, it implies the statement of the theorem.

Proof of Assertion 1. To simplify the notation we write $Q=Q\left(x_{0}\right)$. By the first part of the proof it is sufficient to show that (3.3) holds for $\xi \in A_{K}$. Let $\xi$ be such a point and denote by $M=M(\xi)$ an integer such that

$$
r_{M+1}<\rho_{K}(\xi) \leq r_{M} .
$$


Put

$$
Q_{m}(\xi):=Q \cap S_{m}(\xi), \quad m=0, \ldots, M(\xi), \quad Q_{M+1}=Q \cap B_{r_{M+1}}(\xi) .
$$

By 3.11, 3.12,

$$
K=\bigcup_{m=1}^{M} K_{m}(\xi), \quad Q=\bigcup_{m=0}^{M+1} Q_{m}(\xi) .
$$

By translation we may locate the point $\xi$ at the origin. Therefore in what follows we assume that $\xi=0$ and write $Q_{m}=Q_{m}(0), K_{m}=K_{m}(0)$, etc.

Let $v_{m} \in W_{+}^{2 / q, q^{\prime}}(\partial \Omega)$ be a measure with support in $K_{m}$ and put $v=\sum_{m=1}^{M} v_{m}$ and $\nu_{\epsilon}=\epsilon \nu$ (for $\epsilon>0$ ). Consider the problem

$$
-\Delta u+u^{q}=0 \quad \text { in } Q, \quad u=v_{\epsilon} \quad \text { on } \partial Q .
$$

Let $u$ be the (unique) solution of this problem and let $v$ be the harmonic function with the same boundary data. Then $u<v$ so that

$$
-\Delta u+v^{q}>0 \quad \text { in } Q, \quad u=v_{\epsilon} \quad \text { on } \partial Q .
$$

Hence, by the maximum principle,

$$
u(x) \geq \int_{K} P(x, y) d v_{\epsilon}(y)-\int_{Q} G(x, z)\left(\int_{K} P(z, y) d v_{\epsilon}(y)\right)^{q} d z,
$$

where $P$ and $G$ denote the Poisson and Green kernels in $Q$. Since $u$ vanishes on $\partial Q \backslash K$,

$$
u<u_{K}^{0} \quad \text { in } Q .
$$

From 3.16$)$ we obtain

$$
\begin{aligned}
u(0) & \geq \epsilon \sum_{m=1}^{M} \int_{K_{m}} P(0, y) d v_{m}(y)-\epsilon^{q} \int_{Q} G(0, z)\left(\sum_{m=1}^{M} \int_{K_{m}} P(z, y) d v_{m}(y)\right)^{q} d z \\
& =\epsilon I_{1}-\epsilon^{q} I_{2} .
\end{aligned}
$$

Recall that $c$ or $c_{j}$ denote positive constants which may vary from one formula to another, and which depend only on $q, N, Q$. (In particular, the constants do not depend on $\xi$.) To simplify notation we write $\rho^{\prime}(x)=\rho_{\partial Q}(x)$.

First we observe that

$$
P(x, y) \approx \rho^{\prime}(x)|x-y|^{-N} \quad \forall x \in Q, \forall y \in K .
$$

Hence,

$$
I_{1}=\sum_{m=1}^{M} \int_{K_{m}} P(0, y) d v_{m}(y) \geq c \rho^{\prime}(0) \sum_{m=1}^{M} r_{m}^{-N} v_{m}\left(K_{m}\right) .
$$

In order to estimate $I_{2}$ from above we use the following well-known estimate of the Green kernel:

$$
G(x, z) \approx \frac{\rho^{\prime}(x) \rho^{\prime}(z)|x-z|^{2-N}}{|x-z|^{2}+\rho^{\prime}(x)^{2}+\rho^{\prime}(z)^{2}} \quad \forall x, z \in Q .
$$


As a consequence we find that, for every $z \in B_{r_{j}}(\Xi)$,

$$
G(0, z) \leq c \rho^{\prime}(0) \rho^{\prime}(z) r_{j}^{-N}
$$

Hence

$$
\begin{aligned}
I_{2} & =\int_{Q} G(0, z)\left(\sum_{m=1}^{M} \int_{K_{m}} P(z, y) d v_{m}(y)\right)^{q} d z \\
& =\sum_{j=0}^{M+1} \int_{Q_{j}} G(0, z)\left(\sum_{m=1}^{M} \int_{K_{m}} P(z, y) d v_{m}(y)\right)^{q} d z \\
& \leq c \rho^{\prime}(0) \sum_{j=0}^{M+1} r_{j}^{-N} \int_{Q_{j}}\left(\sum_{m=1}^{M} \int_{K_{m}} P(z, y) d v_{m}(y)\right)^{q} \rho^{\prime}(z) d z .
\end{aligned}
$$

Up to this point the measures $v_{m}$ have not been specified; we define them below. Set

$$
\begin{aligned}
D_{m} & :=\frac{1}{r_{m-1}}\left(Q \cap B_{r_{m-2}}(0)\right)=\left(Q / r_{m-1}\right) \cap B_{2}(0), \\
F_{m} & :=K_{m} / r_{m-1}=\left(K / r_{m-1}\right) \cap S_{1}(0), \\
F_{m}^{*} & :=\left(K / r_{m-1}\right) \cap B_{1 / 2}(0), \quad m=1, \ldots, M .
\end{aligned}
$$

If $\mu_{m}$ is the capacitary measure of $F_{m}$ relative to the capacity $C_{2 / q, q^{\prime}}$ on $\partial D_{m}$ (see [1] Sec. 2.2]) we define $v_{m}$ as follows:

$$
v_{m}(A):=r_{m-1}^{N-1-2 /(q-1)} \mu_{m}\left(A / r_{m-1}\right)
$$

for any Borel set $A \subset K_{m}$. By [1, Thm. 2.2.7], $\mu_{m}$ is a positive measure in $W^{-2 / q, q}\left(\partial D_{m}\right)$ supported in $F_{m}$ and

$$
C_{2 / q, q^{\prime}}\left(F_{m}\right)=\mu_{m}\left(F_{m}\right)=\left\|\mu_{m}\right\|_{W^{-2 / q, q}\left(\partial D_{m}\right)}^{q} .
$$

By 3.27) and 3.28,

$$
v_{m}\left(K_{m}\right)=r_{m-1}^{N-1-2 /(q-1)} \mu_{m}\left(F_{m}\right)=r_{m-1}^{N-1-2 /(q-1)} C_{2 / q, q^{\prime}}\left(F_{m}\right) .
$$

By 3.20 and 3.29,

$$
I_{1} \geq c \rho^{\prime}(0) \sum_{m=1}^{M} r_{m-1}^{-1-2 /(q-1)} C_{2 / q, q^{\prime}}\left(F_{m}\right) .
$$

In addition we have the following inequality:

$$
\sum_{m=1}^{M} r_{m-1}^{-1-2 /(q-1)} C_{2 / q, q^{\prime}}\left(F_{m}\right) \geq c \sum_{m=1}^{M} r_{m-1}^{-1-2 /(q-1)} C_{2 / q, q^{\prime}}\left(F_{m}^{*}\right)
$$

For its proof see Appendix A.

In order to estimate $I_{2}$ we need the following result [15]: If $D$ is a $C^{2}$ bounded domain then, for every measure $\mu \in W^{-2 / q, q}(\partial D)$,

$$
\left\|\mathbb{P}_{D}(\mu)\right\|_{L^{q}\left(D, \rho_{\partial D} d x\right)} \approx\|\mu\|_{W^{-2 / q, q}(\partial D)} .
$$


In general, the constants involved in the relation $\approx$ depend on $q, N$ and $D$. However, a careful examination of the proof of Theorem 2.2 of [15] shows that, for some families of domains, the estimate is uniform. In particular we have:

Assertion 2. Let $\xi \in A_{K}$ and $D_{m}:=\left(Q / r_{m-1}\right) \cap B_{2}(\xi)$ for $1 \leq m \leq M(\xi)$. Then there exists a constant $c$, independent of $\xi$ and $m$, such that

$$
\left.c^{-1}\|\tau\|_{W^{-2 / q, q}} \leq\left\|\mathbb{P}_{D_{m}}(\tau)\right\|_{L^{q}\left(D_{m}, \rho_{m}\right.} d x\right) \leq c\|\tau\|_{W^{-2 / q, q}}
$$

for any measure $\tau \in W_{+}^{-2 / q, q}\left(\partial D_{m}\right)$ with $\operatorname{supp} \tau \subset F_{m}$. Here $\rho_{m}:=\rho_{\partial D_{m}}$.

We continue the proof of Assertion 1, using Assertion 2, whose proof is deferred to the end of this section.

By (3.23),

$$
\begin{aligned}
I_{2} \leq & c \rho^{\prime}(0) \sum_{j=0}^{M+1} r_{j}^{-N} \int_{Q_{j}}\left(\sum_{m=1}^{M} \int_{K_{m}} P(z, y) d v_{m}(y)\right)^{q} \rho^{\prime}(z) d z \\
\leq & c \rho^{\prime}(0) \sum_{j=0}^{M+1} r_{j}^{-N} \int_{Q_{j}} \sum_{m=j-1}^{j+1}\left(\int_{K_{m}} P(z, y) d v_{m}(y)\right)^{q} \rho^{\prime}(z) d z \\
& +c \rho^{\prime}(0) \sum_{j=0}^{M+1} r_{j}^{-N}\left(\sum_{m=1}^{j-2} \sup _{z \in S_{j}} \int_{K_{m}} P(z, y) d v_{m}(y) \rho^{\prime}(z)^{1 / q}\right)^{q}\left|S_{j}\right| \\
& +c \rho^{\prime}(0) \sum_{j=0}^{M+1} r_{j}^{-N}\left(\sum_{m=j+2}^{M} \sup _{z \in S_{j}} \int_{K_{m}} P(z, y) d v_{m}(y) \rho^{\prime}(z)^{1 / q}\right)^{q}\left|S_{j}\right| \\
= & c \rho^{\prime}(0)\left(I_{2,1}+I_{2,2}+I_{2,3}\right)
\end{aligned}
$$

where we put $v_{m}=0$ for $m<1$ or $m>M$. We proceed to estimate each of the three terms on the right hand side of this inequality.

Estimate of $I_{2,1}$. If $1 \leq m \leq M$ and $j-1 \leq m \leq j+1$ then

$$
\begin{aligned}
& Q_{j} / r_{m-1} \subset D_{m}, \\
& P(z, y) \leq c r_{m-1}^{1-N} P_{D_{m}}\left(z^{\prime}, y^{\prime}\right) \quad \forall z \in Q_{j}, \forall y \in K_{m},
\end{aligned}
$$

where $y^{\prime}:=y / r_{m-1}, z^{\prime}:=z / r_{m-1}$ and $c$ is independent of $j, m$. The first relation follows directly from the definition of the domains. To verify the second relation observe that

$$
\rho^{\prime}(z)=\operatorname{dist}(z, \partial Q)=r_{m-1} \operatorname{dist}\left(z^{\prime}, r_{m-1}^{-1} \partial Q\right) \leq 4 r_{m-1} \rho_{m}\left(z^{\prime}\right),
$$

where $\rho_{m}=\rho_{\partial D_{m}}$. Consequently, for every $z \in Q_{j}$ and $y \in K_{m}$,

$$
P(z, y) \leq c_{1} \rho^{\prime}(z)|z-y|^{-N} \leq c_{2} r_{m-1}^{1-N} \rho_{\partial D_{m}}\left(z^{\prime}\right)\left|z^{\prime}-y^{\prime}\right|^{-N} \leq c_{3} r_{m-1}^{1-N} P_{D_{m}}\left(z^{\prime}, y^{\prime}\right) .
$$


Using (3.35) and 3.27) we obtain

$$
\begin{aligned}
& \int_{Q_{j}}\left(\int_{K_{m}} P(z, y) d v_{m}(y)\right)^{q} \rho^{\prime}(z) d z \\
& \leq c \int_{D_{m}}\left(\int_{F_{m}} r_{m-1}^{1-N} P_{D_{m}}\left(z^{\prime}, y^{\prime}\right) r_{m-1}^{N-1-2 /(q-1)} d \mu_{m}\left(y^{\prime}\right)\right)^{q} r_{m-1}^{N+1} \rho_{m}\left(z^{\prime}\right) d z^{\prime} \\
& \leq c r_{m-1}^{N+1-2 q /(q-1)} \int_{D_{m}}\left(\int_{F_{m}} P_{D_{m}}\left(z^{\prime}, y^{\prime}\right) d \mu_{m}\left(y^{\prime}\right)\right)^{q} \rho_{m}\left(z^{\prime}\right) d z^{\prime} \\
& \leq c r_{m-1}^{N-1-2 /(q-1)} C_{2 / q, q^{\prime}}\left(F_{m}\right)
\end{aligned}
$$

By 3.28, 3.33) and 3.36,

$$
\begin{aligned}
I_{2,1} & =\sum_{j=0}^{M+1} r_{j}^{-N} \int_{Q_{j}} \sum_{m=j-1}^{j+1}\left(\int_{K_{m}} P(z, y) d v_{m}(y)\right)^{q} \rho^{\prime}(z) d z \\
& \leq c \sum_{m=1}^{M} r_{m-1}^{-1-2 /(q-1)} C_{2 / q, q^{\prime}}\left(F_{m}\right) .
\end{aligned}
$$

Estimate of $I_{2,2}$. Here $z \in S_{j}, y \in K_{m}$ and $m \leq j-2$. Thus $\rho^{\prime}(z) \approx r_{j},|y-z| \approx r_{m}$ and

$$
P(z, y) \leq c \rho^{\prime}(z)|y-z|^{-N} \leq c r_{m}^{-N} r_{j} .
$$

Hence,

$$
\begin{aligned}
I_{2,2} & =\sum_{j=3}^{M+1} r_{j}^{-N}\left(\sum_{m=1}^{j-2} \sup _{z \in S_{j}} \int_{K_{m}} P(z, y) d v_{m}(y) \rho^{\prime}(z)^{1 / q}\right)^{q}\left|S_{j}\right| \\
& \leq c \sum_{j=3}^{M+1}\left(\sum_{m=1}^{j-2} \sup _{z \in S_{j}} \int_{K_{m}} P(z, y) d v_{m}(y) \rho^{\prime}(z)^{1 / q}\right)^{q} \\
& \leq c \sum_{j=3}^{M+1} r_{j}^{1+q}\left(\sum_{m=1}^{j-2} r_{m}^{-N} v_{m}\left(K_{m}\right)\right)^{q} .
\end{aligned}
$$

Consequently, by 3.29],

$$
\begin{aligned}
I_{2,2} & \leq c \sum_{j=3}^{M+1} r_{j}^{1+q}\left(\sum_{m=1}^{j-2} r_{m-1}^{-1-2 /(q-1)} C_{2 / q, q^{\prime}}\left(F_{m}\right)\right)^{q} \\
& \leq c \sum_{j=3}^{M+1} r_{j}^{1+q}\left(\sum_{m=1}^{j-2} r_{m-1}^{-1-2 /(q-1)} C_{2 / q, q^{\prime}}\left(F_{m}^{*}\right)\right)^{q} .
\end{aligned}
$$

Furthermore,

$$
\sum_{j=3}^{M+1} r_{j}^{1+q}\left(\sum_{m=1}^{j-2} r_{m-1}^{-1-2 /(q-1)} C_{2 / q, q^{\prime}}\left(F_{m}^{*}\right)\right)^{q} \leq c \sum_{m=1}^{M} r_{m-1}^{-1-2 /(q-1)} C_{2 / q, q^{\prime}}\left(F_{m}^{*}\right)
$$

For the proof of this inequality see Appendix B. 
Estimate of $I_{2,3}$. Here $z \in S_{j}, y \in K_{m}$ and $m \geq j+2$. Thus $\rho^{\prime}(z) \approx r_{j},|y-z| \approx r_{j}$ and

$$
P(z, y) \leq c \rho^{\prime}(z)|y-z|^{-N} \leq c r_{j}^{1-N}
$$

Hence

$$
\begin{aligned}
I_{2,3} & =\sum_{j=0}^{M-2} r_{j}^{-N}\left(\sum_{m=j+2}^{M} \sup _{z \in S_{j}} \int_{K_{m}} P(z, y) d v_{m}(y) \rho^{\prime}(z)^{1 / q}\right)^{q}\left|S_{j}\right| \\
& \leq c \sum_{j=0}^{M-2}\left(\sum_{m=j+2}^{M} \sup _{z \in S_{j}} \int_{K_{m}} P(z, y) d v_{m}(y) \rho^{\prime}(z)^{1 / q}\right)^{q} \\
& \leq c \sum_{j=0}^{M-2} r_{j}\left(\sum_{m=j+2}^{M} r_{j}^{1-N} v_{m}\left(K_{m}\right)\right)^{q} \\
& \leq c \sum_{j=0}^{M-2} r_{j}^{1-q(N-1)}\left(\sum_{m=j+2}^{M} r_{m}^{N-1-2 /(q-1)} C_{2 / q, q^{\prime}}\left(F_{m}^{*}\right)\right)^{q} .
\end{aligned}
$$

Put

$$
\theta(r)=\int_{0}^{r} t^{N-2-2 /(q-1)} C_{2 / q, q^{\prime}}\left(\frac{2}{t}\left(K \cap B_{t}\right)\right) d t .
$$

By Lemmas B.1 and B.2 we obtain

$$
\begin{aligned}
& \sum_{j=0}^{M-2} r_{j}^{1-q(N-1)}\left(\sum_{m=j+2}^{M} r_{m}^{N-1-2 /(q-1)} C_{2 / q, q^{\prime}}\left(F_{m}^{*}\right)\right)^{q} \\
& \leq c \sum_{j=0}^{M-2} r_{j}^{1-q(N-1)} \theta\left(r_{j}\right)^{q} \leq c \int_{0}^{\infty} t^{-q(N-1)} \theta(t)^{q} d t .
\end{aligned}
$$

Further, by Hardy's inequality,

$$
\begin{aligned}
\int_{0}^{\infty} t^{-q(N-1)} \theta(t)^{q} d t & \leq c \int_{0}^{\infty} t^{-q(N-2)} \theta^{\prime}(t)^{q} d t \\
& \leq c \int_{0}^{\infty} t^{-2 q /(q-1)} C_{2 / q, q^{\prime}}\left(\frac{2}{t}\left(K \cap B_{t}\right)\right)^{q} d t
\end{aligned}
$$

Combining (3.42) with 3.43) and applying once again Lemmas B.1 and B.2 we obtain

$$
\begin{aligned}
& \sum_{j=0}^{M-2} r_{j}^{1-q(N-1)}\left(\sum_{m=j+2}^{M} r_{m}^{N-1-2 /(q-1)} C_{2 / q, q^{\prime}}\left(F_{m}^{*}\right)\right)^{q} \\
& \quad \leq c \sum_{m=1}^{M} r_{m}^{1-2 q /(q-1)} C_{2 / q, q^{\prime}}\left(F_{m}^{*}\right)^{q} \leq c^{\prime} \sum_{m=1}^{M} r_{m}^{1-2 q /(q-1)} C_{2 / q, q^{\prime}}\left(F_{m}^{*}\right)
\end{aligned}
$$


For the last inequality we used the fact that $C_{2 / q, q^{\prime}}\left(F_{m}^{*}\right)$ is bounded (see 3.26). By 3.41) and 3.44,

$$
I_{2,3} \leq c \sum_{m=1}^{M} r_{m-1}^{-1-2 /(q-1)} C_{2 / q, q^{\prime}}\left(F_{m}^{*}\right) .
$$

Combining (3.34), 3.37), 3.39), 3.40) and 3.45) we obtain

$$
I_{2} \leq c \sum_{m=1}^{M} r_{m-1}^{-1-2 /(q-1)} C_{2 / q, q^{\prime}}\left(F_{m}^{*}\right) .
$$

Finally, by 3.18, 3.30, 3.31 and 3.46,

$$
u(0) \geq \rho^{\prime}(0)\left(c_{1} \epsilon-c_{2} \epsilon^{q}\right) \sum_{m=0}^{M+1} r_{m-1}^{-1-2 /(q-1)} C_{2 / q, q^{\prime}}\left(F_{m}^{*}\right) .
$$

Thus, choosing $\epsilon>0$ sufficiently small we obtain

$$
u(0) \geq c \rho^{\prime}(0) \sum_{m=1}^{M} r_{m-1}^{-1-2 /(q-1)} C_{2 / q, q^{\prime}}\left(F_{m}^{*}\right) .
$$

Proof of Assertion 2. By translation we locate the origin at $\xi$. By an additional rotation we locate the point $\left(0, \ldots, 0, \rho^{\prime}(0)\right)$ at $\sigma(0)(=$ the nearest point to the origin on $\partial Q)$. By the definition of $M=M(\xi)$,

$$
2^{M-1} \rho^{\prime}(0) \leq 2^{M-1} \rho_{K}(0) \leq 1 / 2 .
$$

Therefore, in view of (3.24) and the regularity of $\partial Q$, if $m_{0} \in \mathbb{N}$ is sufficiently large (depending only on $Q$ ) then, for $m_{0}<M$, the domains $D_{m}$ with $m_{0} \leq m \leq M$ "approach" the domain $B_{2}(0) \cap\left\{x: x_{N}<2^{m-1} \rho^{\prime}(0)\right\}$. To make this statement more precise we need some notation:

$$
\begin{aligned}
O_{m}^{r} & :=B_{r}(0) \cap\left\{x: x_{N}<2^{m-1} \rho^{\prime}(0)\right\}, \\
E_{m} & :=\partial D_{m} \cap\left(\frac{1}{r_{m-1}} \partial Q\right) .
\end{aligned}
$$

For every $\alpha>0$ there exists $m_{\alpha}$ such that for every $M>m_{\alpha}$ and every integer $m \in$ $\left[m_{\alpha}, M\right]$, there exists an open neighborhood $U_{m}$ of $\bar{D}_{m}$, a $C^{2}$ diffeomorphism $T_{m}: U_{m} \rightarrow$ $U_{m}^{\prime}$ and a number $d \in(1,1+\alpha)$, independent of $m$, such that

$$
\begin{gathered}
\sup _{m_{\alpha} \leq m \leq M}\left\|T_{m}\right\|_{C^{2}\left(U_{m}\right)} \leq c, \sup _{m_{\alpha} \leq m \leq M}\left\|T_{m}^{-1}\right\|_{C^{2}\left(U_{m}^{\prime}\right)} \leq c, \\
d^{-1}|x| \leq\left|T_{m}(x)\right| \leq d|x|, \\
E_{m}^{\prime}:=T_{m}\left(E_{m}\right) \subset\left\{x: x_{N}=a_{m}\right\},
\end{gathered}
$$

where

$$
\rho^{\prime}(0) / d \leq r_{m-1} a_{m} \leq \rho^{\prime}(0) d
$$


Since $F_{m} \subset E_{m} \cap S_{1}(0)$ we have

$$
F_{m}^{\prime}:=T_{m}\left(F_{m}\right) \subset\left\{x: x_{N}=a_{m}, 1 / 4 d \leq|x| \leq d / 2\right\} .
$$

Let $L_{m}$ be the elliptic operator in $D_{m}^{\prime}$ corresponding to the Laplacian in $D_{m}$ by the transformation $T_{m}$. Let $\tilde{T}_{m}$ denote the mapping induced by $T_{m}$ on the space of Borel measures $\mathfrak{M}\left(\partial D_{m}\right)$ onto $\mathfrak{M}\left(\partial D_{m}^{\prime}\right)$. Then $\tilde{T}_{m}$ maps $W^{-2 / q, q}\left(\partial D_{m}\right)$ continuously onto $W^{-2 / q, q}\left(\partial D_{m}^{\prime}\right)$ and has a continuous inverse. Let $P_{D_{m}}$ (resp. $\left.P_{D_{m}^{\prime}}\right)$ denote the Poisson kernel for $\Delta$ in $D_{m}$ (resp. in $D_{m}^{\prime}$ ) and let $P_{D_{m}^{\prime}}^{L_{m}}$ denote the Poisson kernel for $L_{m}$ in $D_{m}^{\prime}$. If $\tau \in W^{-2 / q, q}\left(\partial D_{m}\right)$ and $\tilde{\tau}:=\tilde{T}_{m}(\tau)$, then

$$
\tilde{u}_{m}:=\mathbb{P}_{D_{m}^{\prime}}^{L_{m}}(\tilde{\tau}), u_{m}:=\mathbb{P}_{D_{m}}(\tau) \Longrightarrow u_{m}=\tilde{u}_{m} \circ \tilde{T}_{m}
$$

and

$$
\mathbb{P}_{D_{m}^{\prime}}^{L_{m}}(\tilde{\tau}) \approx \mathbb{P}_{D_{m}^{\prime}}(\tilde{\tau})
$$

The last relation follows from the equivalence of the Poisson kernels for $L_{m}$ and $\Delta$ in $D_{m}^{\prime}$. In view of the uniform bounds on $T_{m}$, this equivalence relation is uniform with respect to $m$ and $\xi$.

Put

$$
\begin{aligned}
B_{r}^{-}(0) & :=\left\{x \in B_{r}(0): x_{N}<0\right\}, \quad B_{r}^{0}:=\left\{x \in B_{r}(0): x_{N}=0\right\}, \\
S_{m} & :=\left(0,0, \ldots, 0, a_{m}\right), \quad A^{1}:=B_{3 / 2}^{-}(0), \quad A^{2}:=B_{3}^{-}(0) .
\end{aligned}
$$

Then, by 3.52 and 3.49, assuming that $0<\alpha<10^{-1}$,

$$
S_{m}+A^{1} \subset O_{m}^{2 / d} \subset D_{m}^{\prime} \subset O_{m}^{2 d} \subset S_{m}+A^{2} .
$$

By 3.53,

$$
F_{m}^{\prime}=T_{m}\left(F_{m}\right) \subset S_{m}+B_{1}^{0} \subset S_{m}+\partial A^{i}, \quad i=1,2 .
$$

Hence, by 3.56,

$$
\begin{array}{ll}
P_{S_{m}+A^{1}}(x, y) \leq P_{D_{m}^{\prime}}(x, y) & \forall x \in S_{m}+A^{1}, \\
P_{S_{m}+A^{2}}(x, y) \geq P_{D_{m}^{\prime}}(x, y) & \forall x \in D_{m}^{\prime},
\end{array}
$$

for every $y \in S_{m}+B_{1}^{0}$.

If $\tau$ is a bounded Borel measure with support in $F_{m}$, let $\tilde{\tau}_{m}:=\tilde{T}_{m}(\tau)$ and denote by $\tau_{m}^{*}$ the measure on $x_{N}=0$ given by $\tau_{m}^{*}(B)=\tilde{\tau}_{m}\left(B+S_{m}\right)$ for every Borel set $B$. Then, in view of 3.57,

$$
\int_{F_{m}^{\prime}} P_{S_{m}+A^{i}}(x, y) d \tilde{\tau}(y)=\int_{B_{1}^{0}} P_{A^{i}}\left(x^{\prime}, y^{\prime}\right) d \tau^{*}\left(y^{\prime}\right) \quad \forall x \in S_{m}+A^{i},
$$

for $i=1,2$ and $x^{\prime}=x+S_{m}$. By (3.32), if $\tau \in W^{-2 / q, q}\left(\partial D_{m}\right)$ and supp $\tau \subset F_{m}$,

$$
\left\|\tau^{*}\right\|_{W^{-2 / q, q}\left(\partial A^{i}\right)} \approx\left\|\int_{B_{1}^{0}} P_{A^{i}}(\cdot, y) d \tau^{*}(y)\right\|_{L_{b w}^{q}\left(A^{i}\right)}, \quad i=1,2 .
$$


Here for any open set $O \subset \mathbb{R}^{N}, L_{b w}^{q}(O)$ denotes the weighted Lebesgue space $L^{q}$ with weight $\operatorname{dist}(x, \partial O)$.

Combining 3.58, 3.59) and 3.60) we obtain

$$
\begin{aligned}
c_{1}\|\tilde{\tau}\|_{W^{-2 / q, q}\left(S_{m}+\partial A^{1}\right)} & =c_{1}\left\|\tau^{*}\right\|_{W^{-2 / q, q}\left(\partial A^{1}\right)} \\
& \leq\left\|\mathbb{P}_{A^{1}}\left(\tau^{*}\right)\right\|_{L_{b w}^{q}\left(A^{1}\right)} \leq\left\|\mathbb{P}_{D_{m}^{\prime}}(\tilde{\tau})\right\|_{L_{b w}^{q}\left(D_{m}^{\prime}\right)} \leq\left\|\mathbb{P}_{A^{2}}\left(\tau^{*}\right)\right\|_{L_{b w}^{q}\left(A^{2}\right)} \\
& \leq c_{2}\left\|\tau^{*}\right\|_{W^{-2 / q, q}\left(\partial A^{2}\right)}=c_{2}\|\tilde{\tau}\|_{W^{-2 / q, q}\left(S_{m}+\partial A^{2}\right)} .
\end{aligned}
$$

Obviously the constants depend only on $A_{i}$. For measures $\tau \in W^{-2 / q, q}\left(\partial D_{m}\right)$ with support in $F_{m}$,

$$
\|\tilde{\tau}\|_{W^{-2 / q, q}\left(S_{m}+\partial A^{i}\right)} \approx\|\tilde{\tau}\|_{W^{-2 / q, q}\left(S_{m}+B_{5 / 4}^{0}\right)} \approx\|\tau\|_{W^{-2 / q, q}\left(E_{m}\right)}
$$

and, in view of (3.52), the equivalence relations hold with constants independent of $m$ or $\xi$. Hence 3.61) implies 3.33.

\section{A uniqueness result and asymptotic behavior of solutions}

The inequalities established in the previous section are fundamental in the study of positive solutions of the equation (1.2). In this section we present some of the first consequences of these estimates. Further applications, including the full characterization of such solutions in terms of their boundary trace will be presented in a follow-up paper.

Theorem 4.1. Let $\Omega$ be a bounded domain of class $C^{2}$ and $K$ be a compact subset of $\partial \Omega$. As in Section 3 denote by $u_{K}$ the maximal $\sigma$-moderate solution of (1.2) (see (3.2)) and by $U_{K}$ the maximal solution of this equation. Then $U_{K}=u_{K}$.

Proof. By Theorem 3.1. for every point $\xi \in \Omega$,

$$
u_{K}(\xi) \geq c \rho(\xi) \sum_{m=-\ell(\Omega)}^{\infty} r_{m}^{-1-2 /(q-1)} C_{2 / q, q^{\prime}}\left(K_{m}(\xi) / r_{m}\right),
$$

with $K_{m}(\xi)$ as in 3.1 . The constant $c$ depends only on $\Omega, N$ and $q$.

For every $\xi \in \Omega$,

$$
K=\bigcup_{m=-\ell(\Omega)}^{M} K_{m}(\xi), \quad M=M(\xi):=\sup \left\{m \in \mathbb{Z}: K_{m}(\xi) \neq \emptyset\right\},
$$

and consequently,

$$
U_{K}(x) \leq \sum_{m=-\ell(\Omega)}^{\infty} U_{K_{m}(\xi)}(x) \quad \forall x \in \Omega .
$$

To verify this inequality, keep $\xi$ fixed and let $V_{m}$ denote a relatively open neighborhood of $K_{m}(\xi)$ on $\partial \Omega$. Put $V:=\partial \Omega \backslash \bigcup_{m=-\ell(\Omega)}^{M} V_{m}$ and

$$
V_{m}^{\beta}=\left\{x \in \Sigma_{\beta}: \sigma(x) \in V_{m}\right\}, \quad V^{\beta}=\left\{x \in \Sigma_{\beta}: \sigma(x) \in V\right\}
$$


for $\beta \in\left(0, \beta_{0}\right)$ and $-\ell(\Omega) \leq m \leq M(\xi)$. Denote by $v_{m}^{\beta}$ and $v^{\beta}$ the solutions of the boundary value problem

$$
-\Delta v+v^{q}=0 \quad \text { in } \Omega_{\beta}^{\prime}, \quad v=u_{K} \chi_{W} \quad \text { on } \Sigma_{\beta}
$$

with $W=V_{m}^{\beta}$ and $W=V^{\beta}$ respectively. Clearly $\lim _{\beta \rightarrow 0} v^{\beta}=0$ and there exists a sequence $\left\{\beta_{j}\right\}$ decreasing to zero such that $\left\{v_{m}^{\beta_{j}}\right\}$ converges to a solution $v_{m}$ of $(1.2)$ in $\Omega$, for every integer $m \in[-\ell(\Omega), M(\xi)]$. Furthermore $v_{m}$ vanishes on $\partial \Omega \backslash \bar{V}_{m}$ so that $v_{m} \leq u_{\bar{V}_{m}}$. In addition, for every $\beta \in\left(0, \beta_{0}\right)$,

$$
U_{K} \leq \sum_{m=-\ell(\Omega)}^{M} v_{m}^{\beta}+v^{\beta}
$$

so that

$$
U_{K} \leq \sum_{m=-\ell(\Omega)}^{M} v_{m} \leq \sum_{m=-\ell(\Omega)}^{M} U_{\bar{V}_{m}}
$$

Since $U_{K_{m}}=\inf _{K_{m} \subset F} U_{F}(F$ compact subsets of $\partial \Omega$ ), the last inequality implies 4.3 ).

By Theorem 2.1.

$$
U_{K_{m}(\xi)}(x) \leq c \rho_{\partial \Omega}(x) \rho_{m}(x)^{-1-2 /(q-1)} C_{2 / q, q^{\prime}}\left(K_{m}(\xi) / \rho_{m}(x)\right) \quad \forall x \in \Omega,
$$

where $\rho_{m}(x)=\rho_{K_{m}(\xi)}(x)$ and $c$ is a constant depending only on $\Omega, N$ and $q$. Hence, by 4.3,

$$
U_{K}(x) \leq c \sum_{m=-\ell(\Omega)}^{M} \rho_{\partial \Omega}(x) \rho_{m}(x)^{-1-2 /(q-1)} C_{2 / q, q^{\prime}}\left(K_{m}(\xi) / \rho_{m}(x)\right) \quad \forall x \in \Omega .
$$

Since $\rho_{m}(\xi) \in\left[r_{m+1}, r_{m}\right]$ it follows that (see Lemma B.2)

$$
C_{2 / q, q^{\prime}}\left(K_{m}(\xi) / \rho_{m}(\xi)\right) \approx C_{2 / q, q^{\prime}}\left(K_{m}(\xi) / r_{m}\right)
$$

Therefore, by 4.6

$$
U_{K}(\xi) \leq c \rho_{\partial \Omega}(\xi) \sum_{m=-\ell(\Omega)}^{M} r_{m}^{-1-2 /(q-1)} C_{2 / q, q^{\prime}}\left(K_{m}(\xi) / r_{m}\right)
$$

Recall that $\xi$ was an arbitrary point in $\Omega$. Inequalities (4.1) and 4.7) imply that there exists a constant $C$ depending only on $q, N, \Omega$ such that

$$
U_{K}(x) \leq C u_{K}(x) \quad \forall x \in \Omega .
$$

Clearly $u_{K} \leq U_{K}$. If the two solutions are not identical we have

$$
u(x)<U(x) \quad \forall x \in \Omega .
$$

Let $\alpha=1 / 2 C$ and put $v=(1+\alpha) u_{K}-\alpha U_{K}$. Then $\alpha u_{K}<v<u_{K}$. As in [13] we find that $v$ is a supersolution of (1.2). Since $\alpha u_{K}$ is a subsolution it follows that there exists a solution $w$ such that $\alpha u_{K}<w<u_{K}$. If $\mu$ is a positive bounded Borel measure on $\partial \Omega$ 
then $u_{\alpha \mu}$ is the smallest solution which dominates the subsolution $\alpha u_{\mu}$. Hence, if $\mu$ is supported in $K, u_{\alpha \mu}<w$. Therefore

$$
u_{K}=\sup \left\{u_{\alpha \mu}: \mu \in \mathcal{M}_{+}(\partial \Omega)\right\} \leq w .
$$

This contradiction completes the proof.

Theorem 4.2. For every $a \geq 1$ there exists a constant $c(a)>0$, depending also on $q, N, \Omega$, such that, for every $\sigma \in \partial \Omega$,

$$
\begin{aligned}
& \frac{1}{c(a)} \int_{s}^{\beta_{0}} t^{-1-2 /(q-1)} C_{2 / q, q^{\prime}}\left(\frac{1}{t}\left(K \cap \bar{B}_{t}(\sigma)\right)\right) \frac{d t}{t} \leq \frac{U_{K}(x)}{|x-\sigma|} \\
& \leq c(a) \int_{s}^{\beta_{0}} t^{-1-2 /(q-1)} C_{2 / q, q^{\prime}}\left(\frac{1}{t}\left(K \cap \bar{B}_{t}(\sigma)\right)\right) \frac{d t}{t}+O(1)
\end{aligned}
$$

for all $x \in \Omega$ with $s=|x-\sigma| \leq a \rho(x)$. Note that if $\sigma \notin K$ then the integral is bounded.

Proof. The inequality is trivial for $x$ such that $\rho(x) \geq \beta_{0}$. Therefore we assume $\rho(x)<$ $\beta_{0}$. Let $\sigma \in K$. It is sufficient to prove 4.10) (with a constant depending only on $q, N, \Omega$ ) for $x=\sigma-s \nu(\sigma), 0<s<\beta_{0}$. (Recall that $\nu(\sigma)$ is the outward unit normal at $\sigma$.) Indeed, once it is proved in this case, (4.10) follows, by Harnack's inequality, for arbitrary $x \in \Omega$ such that $s=|x-\sigma| \leq a \rho(x)$.

By 4.1, 4.7) and (3.31), if $x=\sigma-s v(\sigma), 0<s<\beta_{0}$,

$$
\begin{aligned}
U_{K}(x) & \approx s \sum_{m=-\ell(\Omega)}^{M} r_{m}^{-1-2 /(q-1)} C_{2 / q, q^{\prime}}\left(K_{m}(x) / r_{m}\right) \\
& \approx s \sum_{m=-\ell(\Omega)}^{M} r_{m}^{-1-2 /(q-1)} C_{2 / q, q^{\prime}}\left(\frac{1}{r_{m}}\left(K \cap B_{r_{m+1}}(x)\right)\right),
\end{aligned}
$$

with $M=M(x)$ as in 4.2). These relations together with Corollary B.3 imply

$$
U_{K}(x) \approx s \int_{2 s}^{\operatorname{diam} \Omega} t^{-1-2 /(q-1)} C_{2 / q, q^{\prime}}\left(\frac{1}{t}\left(K \cap B_{t / 2}(x)\right)\right) \frac{d t}{t} .
$$

Finally, since $B_{t-s}(\sigma) \subset B_{t}(x) \subset B_{3(t-s)}(\sigma)$, a simple computation (using Lemma B.2) yields 4.10 for $x$ as above.

Remark. By [1, Sec. 5.2] (see in particular Cor. 5.2.3 and the first part of the proof of Thm. 5.2.1).

$$
\begin{array}{lll}
C_{2 / q, q^{\prime}}(\gamma E) \approx c \gamma^{N-1-2 /(q-1)} C_{2 / q, q^{\prime}}(E) & \forall \gamma>0, & \text { if } q>q_{c}, \\
C_{2 / q, q^{\prime}}(\gamma E) \leq c \gamma^{N-1-2 /(q-1)} C_{2 / q, q^{\prime}}(E) & \forall \gamma \in(0,1), & \text { if } q=q_{c} .
\end{array}
$$

A set $K$ is said to be $\left(2 / q, q^{\prime}\right)$-thick at a point $\sigma \in \Sigma$ if

$$
J_{q}(\sigma ; K):=\int_{0}^{\beta_{0}}\left(\frac{C_{2 / q, q^{\prime}}\left(K \cap \bar{B}_{t}(\sigma)\right)}{t^{N-1-2 /(q-1)}}\right)^{q-1} \frac{d t}{t}=\infty .
$$


If $J_{q}(\sigma ; K)<\infty$ we say that $K$ is $\left(2 / q, q^{\prime}\right)$-thin at $\sigma$ (see [1]). By [4.13,

$$
\begin{aligned}
& J_{q}(\sigma ; K) \approx \int_{0}^{\beta_{0}} C_{2 / q, q^{\prime}}\left(\frac{1}{t}\left(K \cap \bar{B}_{t}(\sigma)\right)\right)^{q-1} \frac{d t}{t} \quad \text { if } q>q_{c}, \\
& J_{q}(\sigma ; K) \leq \int_{0}^{\beta_{0}} C_{2 / q, q^{\prime}}\left(\frac{1}{t}\left(K \cap \bar{B}_{t}(\sigma)\right)\right)^{q-1} \frac{d t}{t} \quad \text { if } q=q_{c} .
\end{aligned}
$$

It is well known that $K$ is $\left(2 / q, q^{\prime}\right)$-thick, $\left(2 / q, q^{\prime}\right)$-a.e. in $K$, i.e., everywhere except for

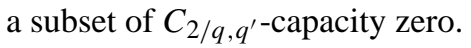

In the next definition we introduce a related notion:

Definition 4.3. A point $\sigma \in \Sigma$ is a $\left(2 / q, q^{\prime}\right)$-concentration point of $K$ if

$$
J_{q}^{*}(\sigma ; K):=\int_{0}^{\beta_{0}} t^{-1-2 /(q-1)} C_{2 / q, q^{\prime}}\left(\frac{1}{t}\left(K \cap \bar{B}_{t}(\sigma)\right)\right) \frac{d t}{t}=\infty .
$$

If the integral is finite we say that $K$ is $\left(2 / q, q^{\prime}\right)$-sparse at $\sigma$.

The function $\theta_{K}(\cdot ; \sigma)$ defined by

$$
\theta_{K}(t ; \sigma):=C_{2 / q, q^{\prime}}\left(\frac{1}{t}\left(K \cap \bar{B}_{t}(\sigma)\right)\right), \quad 0<t \leq 1,
$$

will be called the capacitary distribution of $K$ at $\sigma$.

It is clear that if $K$ is closed (as in our case) then every point at which it is $\left(2 / q, q^{\prime}\right)$ thick (resp. $\left(2 / q, q^{\prime}\right)$-concentrated) belongs to $K$. The following blow-up criterion is an immediate consequence of Theorem 4.2

Corollary 4.4. For every $\sigma \in \Sigma$, either

$$
\lim _{\substack{x \rightarrow \rightarrow_{a}^{\sigma} \\ x \in C_{a}^{\sigma}(\Omega)}} U_{K}(x)|x-\sigma|^{-1}=\infty \quad \text { for every } a \geq 1,
$$

or

$$
\sup _{x \in C_{a}^{\sigma}(\Omega)} U_{K}(x)|x-\sigma|^{-1}<\infty \quad \text { for every } a \geq 1 .
$$

The first case occurs iff $\sigma$ is a $\left(2 / q, q^{\prime}\right)$-concentration point of $K$.

Our next result provides more detailed information on the rate of blow up at points of concentration of $K$.

Theorem 4.5. (a) If $K$ is $\left(2 / q, q^{\prime}\right)$-thick at $\sigma$ then

$$
\int_{0}^{1} U_{K}(\Gamma(t))^{q-1} t d t=\infty
$$

for every curve $\Gamma \in \operatorname{Lip}([0,1], \Omega \cup\{\sigma\})$ such that $\Gamma(0)=\sigma$ and $0<|\Gamma(t)-\sigma| \leq$ $a \rho(\gamma(t))$ for some $a \geq 1$ and every $t \in(0,1]$. Thus 4.17$)$ holds $\left(2 / q, q^{\prime}\right)$-a.e. in $K$. Obviously the integral is finite everywhere outside $K$.

(b) If $K$ is $\left(2 / q, q^{\prime}\right)$-thick at $\sigma$ then it is $\left(2 / q, q^{\prime}\right)$-concentrated at $\sigma$. 
(c) For every $\sigma \in \Sigma$,

$$
\frac{1}{c(a)} s^{-2 /(q-1)} \theta_{K}(s ; \sigma) \leq U_{K}(x) \quad \forall x \in C_{a}^{\Omega}(\sigma),
$$

where $0<s:=|x-\sigma|<1$.

(d) The following asymptotic estimate holds:

$$
\limsup _{\substack{x \rightarrow \sigma \\ x \in C_{a}^{\Omega}}}|x-\sigma|^{2 /(q-1)} U_{K}(x) \stackrel{a}{\approx} \limsup _{s \rightarrow 0} \theta_{K}(s ; \sigma) .
$$

If, in addition, $\theta_{K}(\cdot ; \sigma)$ has a limit as $s \rightarrow 0$, which we denote by $\theta_{K}^{0}(\sigma)$, then

$$
\begin{aligned}
\frac{1}{c(a)} \theta_{K}^{0}(\sigma) \leq & \liminf _{\substack{x \rightarrow \sigma \\
x \in C_{a}^{\Omega}(\sigma)}}|x-\sigma|^{2 /(q-1)} U_{K}(x) \\
\leq & \limsup _{\substack{x \rightarrow \sigma \\
x \in C_{a}^{\Omega}(\sigma)}}|x-\sigma|^{2 /(q-1)} U_{K}(x) \leq c(a) \theta_{K}^{0}(\sigma)
\end{aligned}
$$

for every $a \geq 1$.

Proof. By 4.10, for every $\sigma \in \Sigma$,

$$
\frac{s}{c(a)} \int_{s}^{2 s} t^{-1-2 /(q-1)} \theta_{K}(t ; \sigma) \frac{d t}{t} \leq U_{K}(x)
$$

for every $x \in C_{a}^{\Omega}(\sigma), s=|x-\sigma|<1 / 2$. By Corollary B.3.

$$
\theta_{K}(t ; \sigma) \approx \theta_{K}(s ; \sigma), \quad s<t<2 s .
$$

This implies 4.18). This inequality can be rewritten in the form

$$
\frac{1}{c(a)} \theta_{K}(s ; \sigma)^{q-1} \leq U_{K}(x)^{q-1} s^{2} .
$$

By (4.21) and (4.15), if $x_{s}=\sigma-s v(\sigma)$,

$$
J_{q}(\sigma ; K) \leq c(a) \int_{0}^{\beta_{0}} U_{K}\left(x_{s}\right)^{q-1} s d s
$$

This proves 4.17 in the case $a=1$. Assertion (a) in the general case follows from 4.22) and the Harnack inequality.

If $K$ is $\left(2 / q, q^{\prime}\right)$-thick at $\sigma$ then, by (4.17), for every $\epsilon>0$,

$$
\limsup _{s \rightarrow 0} U_{K}\left(x_{s}\right) s^{2 /(q-1)-\epsilon}=\infty .
$$

By Corollary 4.4 it follows that $\lim U_{K}\left(x_{s}\right) / s=\infty$ and $K$ is $\left(2 / q, q^{\prime}\right)$-concentrated at $\sigma$.

We turn to the proof of the last assertion. Put

$$
V(s):=\int_{s}^{1} t^{-1-2 /(q-1)} \theta_{K}(t ; \sigma) \frac{d t}{t} .
$$


For every $k \geq 1$,

$$
0 \leq V(s)-V(k s)=\int_{s}^{k s} t^{-1-2 /(q-1)} \theta_{K}(t ; \sigma) \frac{d t}{t}
$$

and by Lemma B.2.

$$
\theta_{K}(t ; \sigma) \stackrel{k}{\approx} \theta_{K}(s ; \sigma), \quad s<t<k s .
$$

(The notation $\stackrel{k}{\approx}$ indicates that the constants involved in the equivalence relation depend on $k$.) Hence

$$
0 \leq V(s)-V(k s) \stackrel{k}{\approx} s^{-1-2 /(q-1)} \theta_{K}(s ; \sigma) .
$$

Let $k \geq 1$ and put $x_{s}=\sigma-s v(\sigma)$. Then, by [4.10),

$$
\frac{1}{c} s V(s) \leq U_{K}\left(x_{s}\right) \leq c s(V(s)+O(1)) .
$$

By 4.24, and 4.25,

$$
\begin{aligned}
\frac{1}{c s} U_{K}\left(x_{s}\right) & \leq V(s)+O(1) \\
& \leq V(k s)+c_{k} s^{-1-2 /(q-1)} \theta_{K}(s ; \sigma)+O(1) \\
& \leq \frac{c}{k s} U_{K}\left(x_{k s}\right)+c_{k} s^{-1-2 /(q-1)} \theta_{K}(s ; \sigma)+O(1) .
\end{aligned}
$$

Therefore,

$$
U_{K}\left(x_{s}\right) \leq \frac{c^{2}}{k} U_{K}\left(x_{k s}\right)+c_{k} s^{-2 /(q-1)} \theta_{K}(s ; \sigma)+O(s) .
$$

Applying this inequality with $k^{1+2 /(q-1)}=2 c^{2}$ we obtain

$$
\limsup _{s \rightarrow 0} s^{2 /(q-1)} U_{K}\left(x_{s}\right) \leq c \limsup _{s \rightarrow 0} \theta_{K}(s ; \sigma) .
$$

Combining (4.18) and (4.28) we obtain (4.19) in the case $a=1$. In the general case the inequality follows by an application of Harnack's inequality. Finally, 4.20 is a simple consequence of (4.18) and 4.19).

The following is an immediate consequence of the theorem.

Corollary 4.6. If

$$
\liminf _{s \rightarrow 0} \theta_{K}(s ; \sigma)>0
$$

then, for every $a \geq 1$,

$$
\frac{1}{c(a)}|x-\sigma|^{-2 /(q-1)} \leq U_{k}(x) \leq c(a)|x-\sigma|^{-2 /(q-1)} \quad \text { in } C_{a}^{\Omega}(\sigma) .
$$

If

$$
\lim _{s \rightarrow 0} \theta_{K}(s ; \sigma)=0
$$

then

$$
|x-\sigma|^{2 /(q-1)} U_{K}(x) \rightarrow 0 \quad \text { as } x \rightarrow \sigma \text { non-tangentially. }
$$


Remark. Obviously, condition 4.31) may occur at points where $K$ is $\left(2 / q, q^{\prime}\right)$-thick. Therefore $U_{K}$ may blow up at a rate weaker than the maximal rate even at such points.

\section{Appendix A. Proof of inequality 3.31}

Let $k \in \mathbb{N}$ be a number to be determined later on. Noting that

$$
F_{m}^{*}=\left(r_{k} F_{m+k}^{*}\right) \cup \bigcup_{j=0}^{k-1}\left(r_{j} F_{m+j}\right),
$$

we obtain

$$
\sum_{j=0}^{k-1} C_{2 / q, q^{\prime}}\left(r_{j} F_{m+j}\right) \geq C_{2 / q, q^{\prime}}\left(F_{m}^{*}\right)-C_{2 / q, q^{\prime}}\left(r_{k} F_{m+k}^{*}\right) .
$$

By the argument employed in the first part of the proof of [1, Thm. 5.2.1] (see in particular inequality (5.2.3))

$$
C_{2 / q, q^{\prime}}(t E) \leq A t^{N-1-2 /(q-1)} C_{2 / q, q^{\prime}}(E) \quad \forall t \in(0,1],
$$

for every $q \geq q_{c}=(N+1) /(N-1)$ and every compact set $E \subset \partial Q$, with the constant $A$ depending only on $q, N, \partial Q$. (Recall that, for $q \geq q_{c}, 2 q^{\prime} / q=2 /(q-1) \leq N-1$.) Consequently, by A.1.,

$$
\begin{aligned}
& C_{2 / q, q^{\prime}}\left(F_{m}^{*}\right)-A r_{k}^{N-1-2 /(q-1)} C_{2 / q, q^{\prime}}\left(F_{m+k}^{*}\right) \\
& \leq A \sum_{j=0}^{k-1} r_{j}^{N-1-2 /(q-1)} C_{2 / q, q^{\prime}}\left(F_{m+j}\right) \quad \text { for } m=1, \ldots, M .
\end{aligned}
$$

Now choose $k$ sufficiently large so that $a:=A r_{k}^{N-1}<1$. Recall that $F_{M+j}=F_{M+j}^{*}=\varnothing$ for $j \geq 1$. Clearly

$$
\begin{aligned}
(1- & a) \sum_{m=1}^{M} r_{m-1}^{-1-2 /(q-1)} C_{2 / q, q^{\prime}}\left(F_{m}^{*}\right) \\
\leq & \sum_{m=1}^{M}\left(r_{m-1}^{-1-2 /(q-1)} C_{2 / q, q^{\prime}}\left(F_{m}^{*}\right)-a r_{m+k-1}^{-1-2 /(q-1)} C_{2 / q, q^{\prime}}\left(F_{m+k}^{*}\right)\right) .
\end{aligned}
$$

Further, by (A.3) and A.4,

$$
\begin{aligned}
& \sum_{m=1}^{M} r_{m-1}^{-1-2 /(q-1)} C_{2 / q, q^{\prime}}\left(F_{m}^{*}\right) \leq A^{\prime} \sum_{m=1}^{M} r_{m-1}^{-1-2 /(q-1)} \sum_{j=0}^{k-1} r_{j}^{N-1-2 /(q-1)} C_{2 / q, q^{\prime}}\left(F_{m+j}\right) \\
& =A^{\prime} \sum_{j=0}^{k-1} r_{j}^{N} \sum_{m=1}^{M} r_{m+j-1}^{-1-2 /(q-1)} C_{2 / q, q^{\prime}}\left(F_{m+j}\right) \leq k A^{\prime} \sum_{m=1}^{M} r_{m-1}^{-1-2 /(q-1)} C_{2 / q, q^{\prime}}\left(F_{m}\right),
\end{aligned}
$$

where $A^{\prime}=A /(1-a)$. This proves 3.31). 


\section{Appendix B. Proof of inequality (3.40)}

We start with the following elementary lemma.

Lemma B.1. Let $\varphi_{i}:(0, \infty) \rightarrow[0, \infty), i=1,2$, be measurable functions satisfying the following conditions. There exists a constant $A$ such that, for every $\alpha \in[1 / 2,2]$ and every $t>0$,

$$
A^{-1} \varphi_{2}(t) \leq \varphi_{2}(\alpha t) \leq A \varphi_{2}(t)
$$

and either

$$
A^{-1} \varphi_{1}(t / 2) \leq \varphi_{1}(\alpha t) \leq A \varphi_{1}(2 t)
$$

or

$$
A \varphi_{1}(t / 2) \geq \varphi_{1}(\alpha t) \geq A^{-1} \varphi_{1}(2 t)
$$

Then there exists a constant $c>0$ such that, for every $i, k \in \mathbb{N}, i<k$, the function $\varphi:=\varphi_{1} \varphi_{2}$ satisfies either

$$
c^{-1} \sum_{j=i}^{k} \varphi\left(r_{j+1}\right) \leq \int_{r_{k+1}}^{r_{i}} \varphi(t) \frac{d t}{t} \leq c \sum_{j=i}^{k} \varphi\left(r_{j}\right),
$$

or

$$
c \sum_{j=i}^{k} \varphi\left(r_{j+1}\right) \geq \int_{r_{k+1}}^{r_{i}} \varphi(t) \frac{d t}{t} \geq c^{-1} \sum_{j=i}^{k} \varphi\left(r_{j}\right),
$$

according to which of the conditions (B.2) holds.

Proof. By B.1], B.2a, and the mean value theorem there exists $\tau_{j} \in\left[r_{j+1}, r_{j}\right]$ such that

$$
\frac{1}{2 A^{2}} \varphi\left(r_{j+1}\right) \leq \int_{r_{j+1}}^{r_{j}} \varphi(t) \frac{d t}{t}=r_{j+1} \varphi\left(\tau_{j}\right) / \tau_{j} \leq A^{2} \varphi\left(r_{j}\right) .
$$

This implies B.3a. The second case is similarly verified.

Lemma B.2. For every compact set $F \subset \partial Q$ and every positive number $\tau$,

$$
C_{2 / q, q^{\prime}}(\alpha F) \leq A(\tau) C_{2 / q, q^{\prime}}(F) \quad \forall \alpha \in(0, \tau],
$$

where the constant $A(\tau)$ depends only on $q, N, \partial Q$ and $\tau$ if $q=q_{c}$, and $A(\tau)=$ $A_{0} \tau^{N-1-2 /(q-1)}$, with $A_{0}$ independent of $\tau$, if $q>q_{c}$.

Remark. Recall that $C_{2 / q, q^{\prime}}(F)$ denotes capacity relative to $\partial Q$ while $C_{2 / q, q^{\prime}}(\alpha F)$ denotes capacity relative to $\alpha \partial Q$. 
Proof. Since $\partial Q$ can be covered by a finite number of coordinate patches it is sufficient to prove the inequality in the case that $F$ is contained in a coordinate patch. Thus there exists a $C^{2}$ diffeomorphism of a relative neighborhood $U$ of $F$ onto an open set $U^{\prime}$ in $\mathbb{R}^{N-1}$. Denote this diffeomorphism by $T$ and let $T_{\alpha}$ denote the induced mapping on $\alpha U$ :

$$
T_{\alpha}(\alpha x)=\alpha T(x) \quad \forall x \in U .
$$

Thus $T_{\alpha}(\alpha F)=\alpha T(F)$ and the Lipschitz constant for $T_{\alpha}$ is the same as that for $T$. Since

$$
C_{2 / q, q^{\prime}}(F) \approx C_{2 / q, q^{\prime}}(T(F)), \quad C_{2 / q, q^{\prime}}(\alpha F) \approx C_{2 / q, q^{\prime}}\left(T_{\alpha}(\alpha F)\right),
$$

it is sufficient to prove $\mathrm{B.5}$ ) in the case that $F \subset B_{1} \cap \mathbb{R}^{N-1}$ and the capacity is relative to $\mathbb{R}^{N-1}$. If $q>q_{c}$ the inequality follows immediately from [1, Cor. 5.2.3]. If $q=q_{c}$ we first apply [1, Thm. 5.2.1] to obtain

$$
C_{2 / q, q^{\prime}}(\tau F) \leq C_{\tau} C_{2 / q, q^{\prime}}(F),
$$

where $C_{\tau}$ depends only on $N$ and $\tau$. Next we observe that the first part of the proof of [1. Thm. 5.2.1] and in particular inequality (5.2.3) implies that, for every compact set $E \subset \mathbb{R}^{N-1}$,

$$
C_{2 / q, q^{\prime}}(\gamma E) \leq C^{\prime} \gamma^{N-1-2 /(q-1)} C_{2 / q, q^{\prime}}(E) \quad \forall \gamma \in(0,1]
$$

where $C^{\prime}$ is a constant depending only on $q, N$. This is valid for $1<q \leq q_{c}$. Combining the last two inequalities we obtain (B.5).

Corollary B.3. Let $K$ be a compact subset of $\partial Q$ and put

$$
\phi(t)=C_{2 / q, q^{\prime}}\left(\frac{1}{t}\left(K \cap B_{t / 2}\right)\right)=C_{2 / q, q^{\prime}}\left(\frac{1}{t} K \cap B_{1 / 2}\right) \quad \forall t>0 .
$$

Then $\phi$ satisfies (B.2a) and, for every $a \in \mathbb{R}$ and $i, k \in \mathbb{N}, i<k$,

$$
\frac{1}{c} \sum_{m=i+1}^{k} r_{m}^{a} \phi\left(r_{m}\right) \leq \int_{r_{k}}^{r_{i}} t^{a} \phi(t) \frac{d t}{t} \leq c \sum_{m=i+1}^{k} r_{m-1}^{a} \phi\left(r_{m-1}\right),
$$

where $c$ is a constant depending on $a, q, Q$.

Proof. If $\alpha \in[1 / 2,2]$ then, by Lemma B.2.

$$
\begin{aligned}
\phi(\alpha t) & \geq c C_{2 / q, q^{\prime}}\left(\frac{1}{t}\left(K \cap B_{\alpha t / 2}\right)\right) \\
& \geq c C_{2 / q, q^{\prime}}\left(\frac{1}{t}\left(K \cap B_{t / 4}\right)\right) \geq c C_{2 / q, q^{\prime}}\left(\frac{2}{t}\left(K \cap B_{t / 4}\right)\right)=c \phi(t / 2), \\
\phi(\alpha t) & \leq c C_{2 / q, q^{\prime}}\left(\frac{1}{t}\left(K \cap B_{\alpha t / 2}\right)\right) \\
& \leq c C_{2 / q, q^{\prime}}\left(\frac{1}{t}\left(K \cap B_{t}\right)\right) \leq c C_{2 / q, q^{\prime}}\left(\frac{1}{2 t}\left(K \cap B_{t}\right)\right)=c \phi(2 t) .
\end{aligned}
$$

Thus the function $\phi$ satisfies (B.2a), and consequently, Lemma B.1 implies B.7). 
Proof of (3.40). Let $\phi$ be defined as in (B.6). With this notation

$$
\phi\left(r_{m-1}\right)=C_{2 / q, q^{\prime}}\left(F_{m}^{*}\right) .
$$

Put

$$
\varphi(r, s):=\int_{r}^{s} t^{-1-2 /(q-1)} \phi(t) \frac{d t}{t}, \quad 0<r<s .
$$

By Corollary B.3.

$$
\begin{aligned}
\frac{1}{c} \sum_{m=i+1}^{k} r_{m}^{-1-2 /(q-1)} C_{2 / q, q^{\prime}}\left(F_{m+1}^{*}\right) & \leq \varphi\left(r_{k}, r_{i}\right) \\
& \leq c \sum_{m=i+1}^{k} r_{m-1}^{-1-2 /(q-1)} C_{2 / q, q^{\prime}}\left(F_{m}^{*}\right)
\end{aligned}
$$

for every $i, k \in \mathbb{N}, i<k$. The constant $c$ depends only on $q, N, Q$. Hence

$$
\varphi\left(0, r_{i}\right):=\lim _{r \downarrow 0} \varphi\left(r, r_{i}\right) \leq c \sum_{m=i+1}^{M} r_{m-1}^{-1-2 /(q-1)} C_{2 / q, q^{\prime}}\left(F_{m}^{*}\right) .
$$

Since $\phi$ is bounded,

$$
\varphi(r, \infty) \leq c r^{-1-2 /(q-1)}, \quad r>0 .
$$

Further, by (B.11) and B.10,

$$
\begin{aligned}
& \sum_{j=3}^{M+1} r_{j}^{1+q}\left(\sum_{m=1}^{j-2} r_{m-1}^{-1-2 /(q-1)} C_{2 / q, q^{\prime}}\left(F_{m}^{*}\right)\right)^{q} \\
& \quad \leq c \sum_{j=3}^{M+1} r_{j}^{1+q}\left(\int_{r_{j-3}}^{2} t^{-1-2 /(q-1)} \phi(t) \frac{d t}{t}\right)^{q}=\sum_{j=3}^{M+1} r_{j}^{1+q} \varphi\left(r_{j-3}, 2\right)^{q}
\end{aligned}
$$

By Lemma B.1 (since $\varphi$ is non-increasing, it satisfies B.2b),

$$
\sum_{j=3}^{M+1} r_{j}^{1+q} \varphi\left(r_{j-3}, 2\right)^{q} \leq c \int_{r_{M-1}}^{2} t^{1+q} \varphi(t, 2)^{q} \frac{d t}{t}=c \int_{0}^{2}(t \varphi(t, 2))^{q} d t
$$

By $\mathrm{B} .13$ and $\mathrm{B} .12$,

$$
\begin{aligned}
\int_{0}^{2}(t \varphi(t, 2))^{q} d t & =-\frac{q}{q+1} \int_{0}^{2} t^{q+1} \varphi^{q-1} \varphi^{\prime} d t \\
& \leq-c \int_{0}^{2} \varphi^{\prime} d t=c \varphi(0,2) \leq c \sum_{m=1}^{M} r_{m-1}^{-1-2 /(q-1)} C_{2 / q, q^{\prime}}\left(F_{m}^{*}\right) .
\end{aligned}
$$

Finally B.14 - B.16) imply 3.40. 


\section{Appendix C. Estimates of Poisson kernels}

This appendix is devoted to the derivation of the following theorem.

Theorem C.1. Given $x_{0} \in \mathbb{R}^{N}$ and $0<r$ put

$$
D_{r}\left(x_{0}\right):=\Omega \backslash \bar{B}_{r}\left(x_{0}\right), \quad \Sigma_{r}:=\left\{x \in \mathbb{R}^{N}: \dot{\rho}_{\partial \Omega}(x)=r\right\}
$$

and let $\mathcal{P}_{r}$ denote the Poisson kernel of $-\Delta$ in $D_{r}$. Then there exists a number $m=$ $m(\Omega) \geq 2$ such that, for every $r_{1} \in\left(0, \beta_{0} / 8 m\right), \gamma \in I:=\left[r_{1} / m, r_{1} / 2\right)$ and $x_{0} \in \Sigma_{\gamma}$, the Poisson kernel of $-\Delta$ in $D_{r_{1}}\left(x_{0}\right)$, to be denoted by $\mathcal{P}_{r_{1}}^{x_{0}}$, satisfies the inequality

$$
\mathcal{P}_{r_{1}}^{x_{0}}(x, y) \leq c \frac{\rho_{\partial \Omega}(x) \rho_{\partial \Omega}(y)}{r_{1}|x-y|^{N}} \quad \forall x \in D_{4 r_{1}}\left(x_{0}\right), \quad y \in \partial B_{r_{1}}\left(x_{0}\right) \cap \Omega,
$$

where $c, m$ depend only on $N$ and the $C^{2}$ "norm" of $\partial \Omega$.

We begin with a related estimate whose proof is based on the Harnack inequality up to the boundary (see [2]).

Lemma C.2. Let $G$ be a domain in $\mathbb{R}^{N}$ with compact boundary of class $C^{2}$ and let $\beta^{i}(G), \beta^{e}(G)$ be as in 2.4). Put $\beta_{0}=\min \left(\beta^{i}(G), \beta^{e}(G)\right)$. For every $\gamma \in\left(0, \beta^{e}\right)$ and $\sigma \in \partial G$ put

$$
M_{\gamma}(G):=\left\{\xi \in \mathbb{R}^{N}: \dot{\rho}_{\partial G}(\xi)=-\gamma\right\}, \quad Q_{\gamma}(\sigma):=\sigma+\gamma \nu(\sigma) .
$$

Further set $G_{r}(Q):=G \cap B_{r}(Q)$. Then:

(a) For every $\sigma \in \partial G$ there exists $\bar{\beta} \in\left(0, \beta_{0} / 4\right)$ and an increasing function

$$
R^{\sigma}: I \mapsto(0, \infty), \quad I:=(0, \bar{\beta}),
$$

such that

$$
2 \gamma<R^{\sigma}(\gamma)<\min \left(\beta^{i}+\gamma, \beta^{e}-\gamma\right)
$$

and, for every $\gamma \in I$,

$$
-v(x) \cdot(x-Q) \geq 0 \quad \forall x \in G_{R^{\sigma}(Q)}, Q=Q_{\gamma}(\sigma)
$$

(Recall that $v(x)$ denotes the outward unit normal on $\partial G$ at $\sigma(x)$.)

(b) There exists a constant $C$, depending only on $N, \beta^{e}$ and the Lipschitz constant of $\partial G$, such that, for every $\sigma \in \partial G$ and $\gamma \in I$, the Poisson kernel of $-\Delta$ in $G_{R}(Q)$, where $Q=Q^{\sigma}(\gamma), 2 \gamma<R \leq R^{\sigma}(\gamma)$, satisfies

$$
P_{G_{R}(Q)}(x, y) \leq \frac{C}{\gamma} \frac{\rho_{\partial G}(x) \rho_{\partial G}(y)}{|x-y|^{N}}
$$

for every $x \in G \cap B_{3 R / 4}(Q)$ and $y \in G \cap \partial B_{R}(Q)$. 
Proof. To verify statement (a) it is enough to observe that, if

$$
\bar{\beta}:=-\left(\beta_{0}+1 / 2\right)+\sqrt{\left(\beta_{0}+1 / 2\right)^{2}+\beta_{0}}, \quad R(\gamma):=\sqrt{2 \beta_{0} \gamma+\gamma^{2}},
$$

then the function $R(\cdot)$ on $I$ has all the properties mentioned in (a), for every $\sigma \in \partial G$. However, in general, $R^{\sigma}$ need not tend to zero as $\gamma \rightarrow 0$. For instance, if $G$ is the complement of a ball of radius $\beta_{0}$ and $\bar{\beta}=\beta_{0} / 4$ then the function given by $R(\gamma):=$ $\beta_{0}-\gamma$ satisfies all the conditions stated in (a).

We turn to the proof of (b). We assume (as we may) that $Q$ is the origin and write $B_{r}, G_{r}$ for $B_{r}(0), G_{r}(0)$. The conditions on $x, y$ imply that $R / 4 \leq|x-y|<R$. Therefore C.4 is equivalent to

$$
P_{G_{R}}(x, y) \leq \frac{C}{\gamma R^{N}} \rho_{\partial G}(x) \rho_{\partial G}(y)
$$

where $\gamma=\operatorname{dist}(0, G)$.

For any bounded $C^{2}$ domain $D$ with compact $C^{2}$ boundary and any positive $\alpha$,

$$
P_{D}(x, y)=\alpha^{N-1} P_{\alpha D}(\alpha x, \alpha y) \text {. }
$$

Let $\gamma \in I$ and $R>2 \gamma$ be given. In order to prove that C.5 holds in $G_{R}$ with $\operatorname{dist}(0, G)$ $=\gamma$, it is sufficient to prove that it holds in $\left(\frac{1}{R} G\right)_{1}$ with $\operatorname{dist}\left(\frac{1}{R} G, 0\right)=\gamma / R$ :

$$
P_{\left(\frac{1}{R} G\right)_{1}}(X, Y) \leq \frac{C R}{\gamma} \rho_{\frac{\partial G}{R}}(X) \rho_{\frac{\partial G}{R}}(Y)
$$

for every $X \in\left(\frac{1}{R} G\right) \cap B_{3 / 4}$ and $Y \in\left(\frac{1}{R} G\right) \cap \partial B_{1}$. Indeed, for $x=R X, y=R Y$,

$$
\begin{aligned}
P_{G_{R}}(x, y) & =R^{1-N} P_{\left(\frac{1}{R} G\right)_{1}}(x / R, y / R) \\
& \leq \frac{C}{\gamma} R^{2-N} \rho_{\frac{\partial G}{R}}(x / R) \rho_{\frac{\partial G}{R}}(y / R) \\
& =\frac{C}{\gamma} R^{-N} \rho_{\partial G}(x) \rho_{\partial G}(y) .
\end{aligned}
$$

Note that if C.3 holds in $G_{R}$, relative to $Q=0$, then it also holds in $\left(\frac{1}{R} G\right)_{1}$ relative to the origin.

We turn to the proof of $C .5$ assuming that $\gamma=\operatorname{dist}(0, G) \in(0,1 / 2), R=1$ and $\beta_{0}>2$.

We start with the construction of a certain superhamonic function in $G_{1}=G \cap B_{1}$, vanishing on the boundary, which will be used in our estimate of the Poisson kernel. Let $h, \eta(\cdot ; M, \gamma)$ be solutions of

$$
-\Delta h=1 \quad \text { in } B_{1}, \quad h=0 \quad \text { on } \partial B_{1},
$$

and

$$
-\Delta \eta=1 \quad \text { in } B_{M} \backslash B_{\gamma}, \quad \eta=0 \quad \text { on } \partial B_{\gamma}, \quad \eta=1 \quad \text { on } \partial B_{M},
$$

where $M>\gamma$. Then both $h$ and $\eta$ are radially symmetric, the first decreasing and the 
second increasing with respect to $r=|x|$. Clearly, for every $\alpha>0, \eta(x ; M, \gamma)=$ $\eta(\alpha x ; \alpha M, \alpha \gamma)$. In addition, if $M^{\prime}>M$ then $\eta\left(\cdot ; M^{\prime}, \gamma\right)<1$ on $\partial B_{M}$ and consequently

$$
\eta\left(x ; M^{\prime}, \gamma\right) \leq \eta(x ; M, \gamma) .
$$

Therefore, if $\gamma \in(0,1)$,

$$
\begin{aligned}
& \eta(x ; M, \gamma)=\eta(x / \gamma ; M / \gamma, 1) \quad \forall x \in B_{M} \backslash \bar{B}_{\gamma} \\
& \left.\partial_{r} \eta(\cdot ; M, \gamma)\right|_{\partial B_{\gamma}}=\left.\frac{1}{\gamma} \partial_{r} \eta(\cdot ; M / \gamma, 1)\right|_{\partial B_{1}} \leq\left.\frac{1}{\gamma} \partial_{r} \eta(\cdot ; M, 1)\right|_{\partial B_{1}},
\end{aligned}
$$

where $\partial_{r}$ denotes differentiation in the radial direction on the indicated sphere. For $|x|=r$, let $h_{0}(r):=h(x), \eta_{\gamma}(r):=\eta(x ; 4, \gamma)$.

For every $x \in G_{1}$, put

$$
\tilde{\eta}(x):=\inf \left\{\eta(x-P): P \in M_{\gamma}(G), \sigma(P) \in \partial G \cap B_{1}\right\} .
$$

Then

$$
-\Delta \tilde{\eta} \geq 1 \quad \text { in } G_{1}=G \cap B_{1}, \quad \tilde{\eta}=0 \quad \text { on } \partial G \cap B_{1},
$$

and

$$
\tilde{\eta}(x)=\eta\left(x-P_{x}\right) \quad \text { with } \quad P_{x}=\sigma(x)+\gamma \nu(x) \quad \forall x \in G_{1},
$$

where $v(x)=v_{G}(x)$ denotes the outward unit normal on $\partial G$ at the point $\sigma(x)$. The first statement is obvious since, for every $y \in \partial G \cap B_{1}, P_{y} \in M_{\gamma}(G)$ and $\eta\left(y-P_{y}\right)=0$. The second statement follows from the fact that $\left|x-P_{x}\right| \leq|x-P|$ for every $P \in M_{\gamma}(G)$ such that $\sigma(P) \in \partial G \cap B_{1}$.

Since $\sigma(\cdot)$ and $v(\cdot)$ are in $C^{1}\left((\partial G)^{\beta_{0}}\right)$, the mapping $x \mapsto P_{x}$ is in $C^{1}\left(G_{1}\right)$. Hence $\tilde{\eta} \in C^{1}\left(G_{1}\right)$ and

$$
\begin{aligned}
\partial_{j} \tilde{\eta}(x) & =\eta_{0}^{\prime}\left(\left|x-P_{x}\right|\right) \frac{x-P_{x}}{\left|x-P_{x}\right|} \cdot \partial_{j}\left(x-P_{x}\right) \\
& =\frac{\eta_{0}^{\prime}\left(\left|x-P_{x}\right|\right)}{2\left|x-P_{x}\right|} \partial_{j}\left|x-P_{x}\right|^{2}=\eta_{0}^{\prime}\left(\left|x-P_{x}\right|\right) \partial_{j}\left|x-P_{x}\right| .
\end{aligned}
$$

By $\left[\right.$ C.15], $\left|x-P_{x}\right|=\gamma+\rho_{\partial G}(x)$. Hence

$$
\nabla \tilde{\eta}(x)=\eta_{0}^{\prime}\left(\left|x-P_{x}\right|\right) \nabla \rho_{\partial G}(x) .
$$

If $v:=h \tilde{\eta}$,

$$
\begin{aligned}
-\Delta v & =-(\Delta h) \tilde{\eta}-h \Delta \tilde{\eta}-2 \nabla h \cdot \nabla \tilde{\eta} \\
& \geq \tilde{\eta}+h-2 h_{0}^{\prime}(|x|) \eta_{\gamma}^{\prime}\left(\left|x-P_{x}\right|\right)(x /|x|) \cdot \nabla \rho_{\partial G}(x) .
\end{aligned}
$$

Now $h_{0}^{\prime} \leq 0, \eta_{\gamma}^{\prime} \geq 0$ and $\nabla \rho_{\partial G}(x)$ is in the direction of $-v(x)$. Therefore, by (C.3),

$$
-\Delta v \geq 0 \quad \text { in } G_{1}, \quad v=0 \quad \text { on } \partial G_{1} .
$$

The vanishing on the boundary follows from (C.14) and C.9. 
We turn to the proof of C.5 with $R=1$. By the Harnack inequality up to the boundary (see [2, p. 623]), there exists a constant $C$ which depends only on $N$ and the Lipschitz constant of $\partial G \cap B_{1}$ such that, if $w_{1}, w_{2}$ are positive harmonic functions in $G_{1}$, vanishing on $\partial G \cap B_{1}$,

$$
w_{1}(x) / w_{2}(x) \leq C w_{1}(\xi) / w_{2}(\xi) \quad \forall x, \xi \in \bar{G}_{3 / 4} .
$$

Pick $a, y \in G \cap \partial B_{1}$ and $\xi \in G \cap \partial B_{3 / 4}$ such that $\sigma(a)=\sigma(\xi)$. Applying C.20 with $w_{1}:=P_{G_{1}}(\cdot, y), w_{2}:=P_{G_{1}}(\cdot, a)$ we have

$$
P_{G_{1}}(x, y) \leq C P_{G_{1}}(x, a) \frac{P_{G_{1}}(\xi, y)}{P_{G_{1}}(\xi, a)} \quad \forall x \in \bar{G}_{3 / 4}
$$

We estimate each of the factors on the right hand side.

Since $|a-\xi|=1 / 4$ and $B_{1 / 4}(\xi) \subset G_{1}$ it follows that

$$
P_{G_{1}}(\xi, a) \geq P_{B_{1 / 4}}(0, a-\xi)=c_{1},
$$

where $c_{1}$ is a positive number depending only on $N$.

Let $T_{a}$ denote the half space tangent to $B_{1}$ at $a$, which contains $B_{1}$. Then $G_{1} \subset$ $T_{a} \backslash B_{r}\left(Q^{\sigma}(r)\right):=T_{a, r}^{\sigma}, r:=\min \left(\beta^{e} / 2,2^{-4}\right)$, for every $\sigma \in \partial G \cap B_{1}$. Consequently,

$$
P_{T_{a, r}^{\sigma}}(x, a) \geq P_{G_{1}}(x, a) \quad \forall x \in G_{1} .
$$

By Hopf's lemma, there exists a constant $c_{2}$ depending only on $N$ and $\beta^{e}$ such that

$$
P_{T_{a, r}^{\sigma}}(x, a) \leq c_{2}|x-\sigma| \quad \forall x \in G \cap B_{3 / 4} .
$$

Hence,

$$
P_{G_{1}}(x, a) \leq P_{T_{a, r}^{\sigma(x)}}(x, a) \leq c_{2} \rho_{\partial G}(x) \quad \forall x \in B_{3 / 4} .
$$

For the estimate of $P_{G_{1}}(\xi, y), y \in G \cap \partial B_{1}$, we use the superharmonic function previously constructed. Since $B_{1} \subset B_{4}(\xi)$ it follows that, for every $z \in G_{1} \backslash\{\xi\}$,

$$
\mathcal{G}_{G_{1}}(\xi, z) \leq \mathcal{G}_{B_{4}(\xi)}(\xi, z)=\mathcal{G}_{B_{4}}(0, z-\xi)
$$

where $\mathcal{G}_{D}$ denotes the Green function of $-\Delta$ in $D$. For $\epsilon \in(0,1 / 8)$, put

$$
A_{\epsilon}:=\max _{|\zeta|=\epsilon} \mathcal{G}_{B_{4}}(0, \zeta), \quad A_{\epsilon}^{\prime}=\min _{|\zeta|=\epsilon} v(\xi+\zeta)
$$

Since $0<v<1, A_{\epsilon}^{\prime} \leq 1$. Let $\epsilon_{0}:=\max \left\{\epsilon \in(0,1 / 8): A_{\epsilon} \geq 1\right\}$ and put $c_{3}:=A_{\epsilon_{0}} / A_{\epsilon_{0}}^{\prime}$. Clearly $\epsilon_{0}$ depends only on $N$ and

$$
\mathcal{G}_{G_{1}}(\xi, z) \leq c_{3} v(z) \quad \forall z \in \partial B_{\epsilon_{0}}(\xi)
$$

The function $c_{3} v$ is superharmonic in $G_{1}$; therefore, by the maximum principle (recall that $v=0$ on $\partial G_{1}$ ),

$$
\mathcal{G}_{G_{1}}(\xi, z) \leq c_{3} v(z) \quad \text { in } G_{1} \backslash B_{\epsilon_{0}}(\xi)
$$


This implies

$$
P_{G_{1}}(\xi, y)=-\partial_{\mathbf{n}} \mathcal{G}_{G_{1}}(\xi, y) \leq-c_{3} \partial_{\mathbf{n}} v(y) \quad \forall y \in G \cap \partial B_{1},
$$

where $\mathbf{n}=y /|y|$. Using (C.17) we obtain

$$
\begin{aligned}
-\partial_{\mathbf{n}} v(y) & =-h_{0}^{\prime}(|y|) \tilde{\eta}(y)-h(y) \partial_{\mathbf{n}} \tilde{\eta}(y) \\
& =-h_{0}^{\prime}(1) \eta\left(y-P_{y}\right)-h(y) \eta_{\gamma}^{\prime}\left(\left|y-P_{y}\right|\right) \mathbf{n} \cdot \nabla \rho_{\partial G}(y) \\
& \leq-h_{0}^{\prime}(1) \eta\left(y-P_{y}\right) .
\end{aligned}
$$

The last inequality follows from the fact that $\eta_{\gamma}^{\prime} \geq 0$ and C.3 which implies that $\mathbf{n} \cdot \nabla \rho_{\partial G}(y) \geq 0$. Since $\eta_{\gamma}(\gamma)=0$ and $\eta_{\gamma}$ is increasing and concave,

$$
\eta_{\gamma}\left(\left|y-P_{y}\right|\right)=\eta_{\gamma}\left(\gamma+\rho_{\partial G}(y)\right) \leq \eta_{\gamma}^{\prime}(\gamma) \rho_{\partial G}(y) .
$$

By (C.26)-(C.28) and (C.12],

$$
P_{G_{1}}(\xi, y) \leq \frac{c_{4}}{\gamma} \rho_{\partial G}(y),
$$

where $c_{4}$ depends only on $N$. Finally, (C.21)-C.23) and (C.29) imply (C.4).

Remark. From the proof of the lemma it is clear that the result is of a local nature. In addition, if the $C^{2}$ condition on the boundary is satisfied in a relatively open neighborhood of a point $S \in \partial G$, say $\Gamma_{1}(S)$, then (C.4) holds for all points $Q \in \bar{G}^{c} \cap B_{1 / 2}(S)$ sufficiently close to the boundary of $G$. In this case the relevant values of $\beta^{e}$ and $\beta^{i}$ are those associated with the $C^{2}$ "norm" of $\Gamma_{3 / 4}(S)$.

The following is an immediate consequence of the lemma:

Corollary C.3. If $G$ is a bounded convex domain with $C^{2}$ boundary then (C.4) holds for every $\gamma \in\left(0, \beta_{0}(G) / 3\right)$ and $2 \gamma<R<\gamma+\beta_{0}$ and the constant depends only on $N$ and the Lipschitz constant of $G$. In particular if $G$ is a ball of radius $\beta_{0}$ then the constant depends only on $N$. If $G$ is a half space then (C.4) holds for every $\gamma>0$ and $R>2 \gamma$, the constant depending only on $N$.

Proof. If $G$ is a bounded convex domain with $C^{2}$ boundary then $\beta^{e}=\infty$ and condition (C.3) holds for every $Q \notin \bar{G}$. This implies the statements of the lemma.

Lemma C.4. Let $\Omega=\left\{X \in \mathbb{R}^{N}: X_{1}>0\right\}, \gamma^{*}>0$ and $R^{*} \geq 3 \gamma^{*}$. Then the Poisson kernel of the domain

$$
D^{*}:=D_{R^{*}}(Z)=\Omega \backslash B_{R^{*}}(Z), \quad Z=\left(\gamma^{*}, 0, \ldots, 0\right),
$$

satisfies the inequality

$$
\mathcal{P}_{D^{*}}(X, Y) \leq c \frac{X_{1} Y_{1}}{\gamma^{*}|X-Y|^{N}}
$$

for every $X, Y \in \Omega$ such that $|X-Z|>4 R^{*} / 3$ and $|Y-Z|=R^{*}$, with the constant $c$ depending only on $N$.

Proof. Put

$$
\gamma=\frac{\gamma^{*}}{\left(R^{*}\right)^{2}-\left(\gamma^{*}\right)^{2}}, \quad R:=\frac{R^{*}}{\left(R^{*}\right)^{2}-\left(\gamma^{*}\right)^{2}}, \quad Q_{\gamma}^{0}=(-\gamma, 0,, \ldots, 0) .
$$


By scaling (see (C.6) we may assume that $R^{*}=1$ and $\gamma^{*}<1 / 3$. In this case $0<$ $\gamma<3 / 8$ and $1<R<9 / 8$. The inversion $T$ relative to the sphere $|x|=1$ maps $B_{R}\left(Q_{\gamma}^{0}\right)$ onto the exterior of the ball $B_{R^{*}}(Z)$ and $\Omega$ onto itself. Consequently, $B_{R}\left(Q_{\gamma}^{0}\right) \cap$ $\Omega$ is transformed to $D^{*}$. By Corollary C.3 the Poisson kernel of $D=B_{R}\left(Q_{\gamma}^{0}\right) \cap \Omega$ satisfies inequality (C.5), which in this case is equivalent to C.4. By a straightforward computation, if $Q$ is a bounded domain of class $C^{1}$ then

$$
P_{Q}(x, y)=|x|^{2-N} P_{T(Q)}(X, Y), \quad X=x /|x|^{2}, Y=y /|y|^{2} .
$$

Applying this relation to $D=B_{R}\left(Q_{\gamma}^{0}\right) \cap \Omega$ and $D^{*}=T(D)=D_{R^{*}}(Z)$ and using (C.5), we obtain

$$
P_{D^{*}}(X, Y) \leq|X|^{2-N} \frac{C}{\gamma} x_{1} y_{1}
$$

for every $X, Y$ such that $\left|x-Q_{\gamma}^{0}\right|<3 R / 4$ and $\left|y-Q_{\gamma}^{0}\right|=1$. Since $x_{1}=X_{1} /|X|^{2}$ we obtain

$$
P_{D^{*}}(X, Y) \leq \frac{C}{\gamma}|X|^{-N} X_{1} Y_{1}|Y|^{-2} \text {. }
$$

Since $R^{*}=1,1<R<9 / 8$ and $R / \gamma=R^{*} / \gamma^{*}$ it follows that $1<\gamma / \gamma^{*}<9 / 8$. If $|X-Z|>4 / 3$ and $|Y-Z|=1$ then $|X-Y| \approx|X|,|Y| \approx 1$. Hence

$$
P_{D^{*}}(X, Y) \leq \frac{C}{\gamma^{*}}|X-Y|^{-N} X_{1} Y_{1}
$$

Lemma C.5. Let $R, r_{1}, \gamma_{1}$ be given such that

$$
R / r_{1}:=m>4, \quad 1 / m<\gamma_{1} / r_{1}<1 .
$$

Denote by $\mathbf{e}$ the unit vector in the direction of the positive $x_{1}$-axis, put $\tau_{1}:=2 R+\gamma_{1}$ and

$$
\Omega:=\left\{x \in \mathbb{R}^{N}:|x+R \mathbf{e}|>R\right\}, \quad D^{*}:=\Omega \backslash B_{r_{1}}\left(-\tau_{1} \mathbf{e}\right) .
$$

Then the Poisson kernel of $D^{*}$ satisfies the inequality

$$
P_{D^{*}}(x, y) \leq c m R^{N-1} \frac{\rho_{\partial \Omega}(x) \rho_{\partial \Omega}(y)}{r_{1}|x-y|^{N}}\left(R+\rho_{\partial \Omega}(x)\right)
$$

for every $x, y \in \Omega$ such that $\left|x+\tau_{1} \mathbf{e}\right|>4 r_{1}$ and $\left|y+\tau_{1} \mathbf{e}\right|=r_{1}$, with the constant $c$ depending only on $N$.

Proof. Put $a:=1 / 2 R, \mu:=\tau_{1} / r_{1}=\left(2 R+\gamma_{1}\right) / r_{1}$ and compute $\gamma_{0}$ and $r_{0}$ from the relations

$$
r_{1}=\frac{r_{0}}{\left(a-\gamma_{0}\right)^{2}-r_{0}^{2}}, \quad \tau_{1}=\frac{a-\gamma_{0}}{\left(a-\gamma_{0}\right)^{2}-r_{0}^{2}} .
$$

This yields

$$
r_{0}=\frac{1}{r_{1}\left(\mu^{2}-1\right)}=\frac{\mu}{\tau_{1}\left(\mu^{2}-1\right)}, \quad \gamma_{0}=a-\mu r_{0} .
$$

We note that

$$
\gamma_{0} \geq \frac{1}{16 m^{3} r_{1}}=\frac{r_{1}}{16 m R^{2}} .
$$


Indeed, by C.34,

$$
\gamma_{0}=\frac{1}{\tau_{1}-\gamma_{1}}-\frac{\tau_{1}}{\tau_{1}^{2}-r_{1}^{2}}=\frac{\gamma_{1} \tau_{1}-r_{1}^{2}}{\left(\tau_{1}-\gamma_{1}\right)\left(\tau_{1}^{2}-r_{1}^{2}\right)}
$$

and, by assumption, $\tau_{1} / r_{1}=2 m$ so that $\gamma_{1} \tau_{1}>2 r_{1}^{2}$. Hence

$$
\gamma_{0} \geq \frac{r_{1}^{2}}{\left(\tau_{1}-\gamma_{1}\right)\left(\tau_{1}^{2}-r_{1}^{2}\right)}=\frac{1}{r_{1}} \frac{1}{\left(\mu^{2}-1\right)(\mu-1)},
$$

which implies C.35).

Now consider the domain

$$
D_{0}:=\left\{X \in \mathbb{R}^{N}: X_{1}>-a\right\} \backslash B_{r_{0}}\left(\left(-a+\gamma_{0}\right) \mathbf{e}\right) .
$$

Let $T$ denote the inversion relative to the unit sphere centered at the origin: $T X=$ $X /|X|^{2}$. Then

$$
T(E)= \begin{cases}\Omega & \text { if } E=\left\{X \in \mathbb{R}^{N}: X_{1}>-a\right\}, \\ B_{r_{1}}\left(-\tau_{1} \mathbf{e}\right) & \text { if } E=B_{r_{0}}\left(\left(-a+\gamma_{0}\right) \mathbf{e}\right), \\ B_{r_{2}}\left(-\tau_{2} \mathbf{e}\right) & \text { if } E=B_{2 r_{0}}\left(\left(-a+\gamma_{0}\right) \mathbf{e}\right),\end{cases}
$$

where

$$
r_{2}=\frac{2 r_{0}}{\left(a-\gamma_{0}\right)^{2}-4 r_{0}^{2}}, \quad \tau_{2}=\frac{a-\gamma_{0}}{\left(a-\gamma_{0}\right)^{2}-4 r_{0}^{2}} .
$$

Therefore $T\left(D_{0}\right)=D^{*}$ and

$$
B_{r_{2}}\left(-\tau_{2} \mathbf{e}\right) \subset B_{4 r_{1}}\left(-\tau_{1} \mathbf{e}\right) .
$$

Indeed, by C.34, $\mu=\left(a-\gamma_{0}\right) / r_{0}$ and consequently

$$
\frac{\tau_{2}-\tau_{1}}{r_{1}}=\frac{3 r_{0}\left(a-\gamma_{0}\right)}{\left(a-\gamma_{0}\right)^{2}-4 r_{0}^{2}}=\frac{3 \mu}{\mu^{2}-4} .
$$

If $X=-\tau_{1} \mathbf{e}+4 r_{1} \bar{h}$, where $\bar{h}$ is a unit vector, it follows that

$$
\left|X+\tau_{2} \mathbf{e}\right| \geq 4 r_{1}-\left(\tau_{2}-\tau_{1}\right)=r_{1}\left(4-\frac{3 \mu}{\mu^{2}-4}\right),
$$

and, since $\mu=\tau_{1} / r_{1}>2 m \geq 8$,

$$
r_{1}\left(4-\frac{3 \mu}{\mu^{2}-4}\right) \geq r_{2}=\frac{2}{r_{0}\left(\mu^{2}-4\right)}=\frac{2 r_{1}\left(\mu^{2}-1\right)}{\mu^{2}-4},
$$

which proves C.38.

By Lemma C.4.

$$
P_{D_{0}}(X, Y) \leq c \frac{\left(X_{1}+a\right)\left(Y_{1}+a\right)}{\gamma_{0}|X-Y|^{N}}
$$


for every $X, Y \in\left\{X \in \mathbb{R}^{N}: X_{1}>-a\right\}$ with $\left|X+\left(a-\gamma_{0}\right) \mathbf{e}\right|>2 r_{0}$ and $\left|Y+\left(a-\gamma_{0}\right) \mathbf{e}\right|$ $=r_{0}$. The constant depends only on $N$. By [C.31], as $T\left(D_{0}\right)=D^{*}$,

$$
P_{D^{*}}(x, y)=|x|^{2-N} P_{D_{0}}(X, Y), \quad X=x /|x|^{2}, Y=y /|y|^{2} .
$$

From C.38 C.40 we obtain

$$
P_{D^{*}}(x, y) \leq c \frac{\left(X_{1}+a\right)\left(Y_{1}+a\right)}{\gamma_{0}|X-Y|^{N}}|x|^{2-N}
$$

for every $x, y \in \Omega$ such that $\left|x+\tau_{1} \mathbf{e}\right|>4 r_{1}$ and $\left|y+\tau_{1} \mathbf{e}\right|=r_{1}$. It is easily verified that

$$
|T x-T y|=\frac{|x-y|}{|x||y|} \text {. }
$$

Therefore, by $\overline{C .41}$,

$$
P_{D^{*}}(x, y) \leq c \frac{\left(2 R x_{1}+|x|^{2}\right)\left(2 R y_{1}+|y|^{2}\right)}{\gamma_{0} R^{2}|x-y|^{N}|y|^{2-N}},
$$

for $x, y$ as before. A simple computation yields the identity

$$
|x|^{2}+2 R x_{1}=\delta_{R}(x)\left(2 R+\delta_{R}(x)\right), \quad \delta_{R}(x):=\operatorname{dist}\left(x, \partial B_{R}(-R \mathbf{e})\right) .
$$

Hence (C.43) implies

$$
P_{D^{*}}(x, y) \leq c \frac{\delta_{R}(x) \delta_{R}(y)\left(2 R+\delta_{R}(x)\right)\left(2 R+\delta_{R}(y)\right)}{\gamma_{0} R^{2}|x-y|^{N}|y|^{2-N}} .
$$

If $\left|y+\tau_{1} \mathbf{e}\right|=r_{1}$ then $\delta_{R}(y) \leq 2 r_{1}=2 R / m$ and $R \leq|y| \leq 3 R$. Therefore, by (C.43) and C.35,

$$
\begin{aligned}
P_{D^{*}}(x, y) & \leq c \frac{\delta_{R}(x) \delta_{R}(y)\left(2 R+\delta_{r}(x)\right)}{\gamma_{0} R^{3-N}|x-y|^{N}} \\
& \leq c m R^{N-1} \frac{\delta_{R}(x) \delta_{R}(y)}{r_{1}|x-y|^{N}}\left(R+\left(\delta_{R}(x)\right)\right),
\end{aligned}
$$

with the constant $c$ depending only on $N$.

Proof of the theorem. Choose $m$ large enough so that if $r_{1} \in\left(0, \beta_{0} / 8 m\right)$ and $x_{0} \in \Omega$ satisfies $\rho_{\partial \Omega}\left(x_{0}\right)=\gamma \in\left[r_{1} / m, r_{1} / 8\right)$ then

$$
\nu(x) \cdot\left(x-x_{0}\right) \geq 0 \quad \forall x \in \partial B_{r_{1}}\left(x_{0}\right) \cap \partial \Omega .
$$

Such a choice of $m$ exists because $\Omega$ is bounded of class $C^{2}$.

Let $r_{1}$ and $x_{0}$ be as above and let $\xi$ be a point in $\partial B_{r_{1}}\left(x_{0}\right) \cap \partial \Omega$. Put $R=m r_{1}$ and $\xi_{0}=R v(\xi)$. Define

$$
B_{R}^{\prime}\left(\xi_{0}\right):=\left\{x \in \mathbb{R}^{N}:\left|x-\xi_{0}\right|>R\right\}, \quad D_{r_{1}}^{*}\left(x_{0} ; \xi_{0}\right):=B_{R}^{\prime}\left(\xi_{0}\right) \backslash \bar{B}_{r_{1}}\left(x_{0}\right) .
$$

Then

$$
\bar{\Omega} \cap \bar{B}_{R}^{\prime}\left(\xi_{0}\right)=\{\xi\}, \quad D_{r_{1}}\left(x_{0}\right) \subset D_{r_{1}}^{*}\left(x_{0}, \xi_{0}\right)
$$


Therefore

$$
\mathcal{P}_{r_{1}}(x, y) \leq P_{D_{r_{1}}^{*}}(x, y) \quad \forall x \in D_{r_{1}}\left(x_{0}\right), y \in \partial B_{r_{1}}\left(x_{0}\right) \cap \Omega .
$$

A simple computation shows that

$$
\gamma \leq \operatorname{dist}\left(x_{0}, \partial B_{R}^{\prime}\left(\xi_{0}\right)\right) \leq \gamma+\frac{c}{R} r_{1}^{2}=\gamma+c r_{1} / m
$$

where $c$ is a constant depending only on $\Omega$ and $\beta_{0}$. Therefore, by choosing $m$ sufficiently large, we guarantee that $\gamma \leq \operatorname{dist}\left(x_{0}, \partial B_{R}^{\prime}\left(\xi_{0}\right)\right) \leq 2 \gamma$. By Lemma C.5.

$$
P_{D_{r_{1}}^{*}}(x, y) \leq C \frac{\rho_{E_{R}}(x) \rho_{E_{R}}(y)}{r_{1}|x-y|^{N}} \leq C \frac{\rho_{E_{R}}(y)}{r_{1}|x-y|^{N-1}}, \quad E_{R}=\partial B_{R}^{\prime}\left(\xi_{0}\right),
$$

for every $x, y \in \Omega$ such that $\left|x-x_{0}\right|>4 r_{1},\left|y-x_{0}\right|=r_{1}$, with $C=c m(1+\operatorname{diam} \Omega) \beta_{0}^{N-1}$. The second inequality follows from the fact that, for any $x, y$ as above,

$$
\rho_{E_{R}}(x) \leq \rho_{D_{r_{1}}^{*}}(x)+2 r_{1} \leq 2|x-y| .
$$

If $y \in \partial B_{r_{1}}\left(x_{0}\right) \cap \Omega$ is a point such that

$$
|y-\xi|=\inf _{\eta \in \partial B_{r_{1}}\left(x_{0}\right) \cap \partial \Omega}|y-\eta|
$$

then $\rho_{E_{R}}(y) \approx|y-\xi| \approx \rho_{\partial \Omega}(y)$ and consequently, by C.46 and C.47),

$$
\mathcal{P}_{r_{1}}(x, y) \leq C \frac{\rho_{\partial \Omega}(y)}{r_{1}|x-y|^{N-1}}
$$

for every $x \in \Omega$ such that $4 r_{1}<\left|x-x_{0}\right|$. For every $y \in \partial B_{r_{1}}\left(x_{0}\right) \cap \Omega$ there exists a nearest point $\xi \in \partial B_{r_{1}}\left(x_{0}\right) \cap \partial \Omega$ (although $\xi$ may not be unique). Since $C$ is independent of $\xi$, it follows that

$$
\mathcal{P}_{r_{1}}(x, y) \leq C \frac{\rho_{\partial \Omega}(y)}{r_{1}|x-y|^{N-1}}
$$

for every $y \in \partial B_{r_{1}}\left(x_{0}\right) \cap \Omega$ and every $x \in \Omega$ such that $4 r_{1}<\left|x-x_{0}\right|$.

By a standard argument, C.48 implies (C.1). Obviously (C.1) holds for pairs of points $(x, y)$ such that $|x-y| \leq 8 \rho_{\partial \Omega}(x)$. Therefore we consider pairs $\left(x^{\prime}, y^{\prime}\right)$ such that

$$
y^{\prime} \in \partial B_{r_{1}}\left(x_{0}\right) \cap \Omega, \quad x^{\prime} \in D_{4 r_{1}}\left(x_{0}\right), \quad \rho_{\partial \Omega}\left(x^{\prime}\right) \leq 1 / 8\left|x^{\prime}-y^{\prime}\right| .
$$

Put

$$
E^{\prime}:=\left\{x \in \Omega:\left|x-\sigma\left(x^{\prime}\right)\right|<\alpha / 2\right\}, \quad \alpha:=\left|x^{\prime}-y^{\prime}\right| .
$$

For $x \in E^{\prime}$, we have $\left|x-y^{\prime}\right| \leq \alpha$. Therefore

$$
\mathcal{P}_{r_{1}}\left(x, y^{\prime}\right) \leq C \frac{\rho_{\partial \Omega}\left(y^{\prime}\right)}{\alpha^{N-1} r_{1}}:=A \quad \forall x \in E^{\prime} .
$$

The function $\mathcal{P}_{r_{1}}\left(\cdot, y^{\prime}\right)$ is harmonic and vanishes on $\partial \Omega$. By Hopf's lemma,

$$
\mathcal{P}_{r_{1}}\left(x, y^{\prime}\right) \leq A \rho_{\partial \Omega}(x) / \alpha \quad \forall x \in \Omega:\left|x-\sigma\left(x^{\prime}\right)\right|<\frac{1}{4}\left|x^{\prime}-y^{\prime}\right| .
$$

In particular

$$
\mathcal{P}_{r_{1}}\left(x^{\prime}, y^{\prime}\right) \leq C \frac{\rho_{\partial \Omega}\left(x^{\prime}\right) \rho_{\partial \Omega}\left(y^{\prime}\right)}{\alpha^{N} r_{1}}
$$




\section{References}

[1] Adams, D. R., Hedberg, L. I.: Function Spaces and Potential Theory. Grundlehren Math. Wiss. 314, Springer (1996) Zbl 0834.46021 MR 97j:46024

[2] Caffarelli, L., Fabes, E., Mortola, S., Salsa, S.: Boundary behavior of nonnegative solutions of elliptic operators in divergence form. Indiana Univ. Math. J. 30, 621-640 (1981) Zbl 0512.35038 MR83c:35040

[3] Dynkin, E. B.: New relations between diffusions and superdifusions and their applications to differential equations. Preprint (see [6])

[4] Dynkin, E. B.: A new inequality for superdiffusions and its applications to nonlinear differential equations. C. R. Acad. Sci. Paris Sér. I (to appear)

[5] Dynkin, E. B.: Absolute continuity results for superdiffusions with applications to differential equations. Preprint.

[6] Dynkin, E. B.: Superdiffusion and Positive Solutions of Nonlinear Partial Differential Equations. Forthcoming book

[7] Dynkin, E. B., Kuznetsov, S. E.: Fine topology and fine trace on the boundary associated with a class of semilinear differential equations. Comm. Pure Appl. Math. 51, 897-936 (1998) Zbl 0934.35049 MR 99f:35046

[8] Dynkin, E. B., Kuznetsov, S. E.: Poisson capacities. Math. Res. Lett. 10, 85-95 (2003) Zbl 1027.31008 MR 2003k:31005

[9] Gmira, A., Véron, L.: Boundary singularities of solutions of some nonlinear elliptic elliptic equations. Duke Math. J. 64, 271-324 (1991) Zbl 0766.35015 MR 93a:35053

[10] Labutin, D. A.: Wiener regularity for large solutions of nonlinear equations. Ark. Mat. 41, 307-339 (2003) Zbl 02057392 MR 2011924

[11] Legall, J. F.: The Brownian snake and solutions of $\Delta u=u^{2}$ in a domain. Probab. Theory Rellated Fields 102, 393-432 (1995) MR 96c:60098

[12] Marcus, M., Véron, L.: Trace au bord des solutions positives d'équations elliptiques et paraboliques non linéaires: Résultats d'existence et d'unicité. C.R. Acad. Sci. Paris Sér. I 323, 603-608 (1996) Zbl 0858.35127 MR 97f:35012

[13] Marcus, M., Véron, L.: The boundary trace of positive solutions of semilinear elliptic equations: the subcritical case. Arch. Rat. Mech. Anal. 144, 201-231 (1998) Zbl 0924.35050 MR 2000a:35077

[14] Marcus, M., Véron, L.: The boundary trace of positive solutions of semilinear elliptic equations: the supercritical case. J. Math. Pures Appl. 77, 481-524 (1998) Zbl 0933.35081 MR 99g:35045

[15] Marcus, M., Véron, L.: Removable singularities and boundary trace. J. Math. Pures Appl. 80, 879-900 (2000) Zbl 01770849 MR 2002j:35124

[16] Marcus, M., Véron, L.: Capacitary estimates of solutions of a class of nonlinear elliptic equations. C. R. Acad. Sci. Paris 336, 913-918 (2003) Zbl 01981310 MR 2004e:35077

[17] Mselati, B.: Classification et représentation probabiliste des solutions positives de $\Delta u=u^{2}$ dans un domaine. Thèse de Doctorat, Univ. Paris 6 (2002)

[18] Mselati, B.: Classification and probabilistic representation of the positive solutions of a semilinear elliptic equation. Mem. Amer. Math. Soc. 168, no. 798 (2004) Zbl 02056300 MR 2031708 